\title{
Elucidating how the saprophytic fungus Aspergillus nidulans uses the plant polyester suberin as carbon source
}

\author{
Isabel Martins ${ }^{1}$, Diego O Hartmann ${ }^{1}$, Paula C Alves ${ }^{1}$, Celso Martins ${ }^{1,2}$, Helga Garcia ${ }^{1}$, Céline C Leclercq ${ }^{3}$, \\ Rui Ferreira', Ji He${ }^{4}$, Jenny Renaut ${ }^{3}$, Jörg D Becker ${ }^{5}$ and Cristina Silva Pereira ${ }^{1,2^{*}}$
}

\begin{abstract}
Background: Lipid polymers in plant cell walls, such as cutin and suberin, build recalcitrant hydrophobic protective barriers. Their degradation is of foremost importance for both plant pathogenic and saprophytic fungi. Regardless of numerous reports on fungal degradation of emulsified fatty acids or cutin, and on fungi-plant interactions, the pathways involved in the degradation and utilisation of suberin remain largely overlooked. As a structural component of the plant cell wall, suberin isolation, in general, uses harsh depolymerisation methods that destroy its macromolecular structure. We recently overcame this limitation isolating suberin macromolecules in a near-native state.

Results: Suberin macromolecules were used here to analyse the pathways involved in suberin degradation and utilisation by Aspergillus nidulans. Whole-genome profiling data revealed the complex degrading enzymatic machinery used by this saprophytic fungus. Initial suberin modification involved ester hydrolysis and $\omega$-hydroxy fatty acid oxidation that released long chain fatty acids. These fatty acids were processed through peroxisomal $\beta$-oxidation, leading to up-regulation of genes encoding the major enzymes of these pathways (e.g. faaB and aoxA). The obtained transcriptome data was further complemented by secretome, microscopic and spectroscopic analyses.

Conclusions: Data support that during fungal growth on suberin, cutinase 1 and some lipases (e.g. AN8046) acted as the major suberin degrading enzymes (regulated by FarA and possibly by some unknown regulatory elements). Suberin also induced the onset of sexual development and the boost of secondary metabolism.
\end{abstract}

Keywords: Aspergillus nidulans, ß-oxidation, Cutinase, Long chain fatty acids, Suberin, Whole-genome profiling

\section{Background}

Plant lipid polymers, particularly cutin and suberin, are the third most abundant of the plant polymers [1], yet the least understood since the underlying polyester structure remains partially unresolved. High recalcitrance is an inherent property of their molecular structure and hallmark monomers are often identified in soils [2,3]. Filamentous fungi are key recyclers and compose nearly $75 \%$ of the soil microbial biomass [4] but their role in the turnover (biodegradation) of plant polyesters remains largely overlooked [5]. Suberin is a structural component of the

\footnotetext{
* Correspondence: spereira@itqb.unl.pt

'Instituto de Tecnologia Química e Biológica, Universidade Nova de Lisboa,

Av. da República, 2780-157 Oeiras, Portugal

Instituto de Biologia Experimental e Tecnológica (BBET), Av. da República, 2781-901 Oeiras, Portugal

Full list of author information is available at the end of the article
}

secondary cell walls in specialised tissues, namely in the phellem of tree barks and subterranean organs $[5,6]$ and in the endodermis of roots [7]. Cutin, together with waxes (viz. cuticle), covers the cell walls in the epidermis of aerial tissues [8,9]. Biosynthesis of either suberin or cutin is developmentally regulated and triggered as response to infection or wounding, among other challenges [8-13]. These lipid hydrophobic barriers constrain apoplastic water and solute translocation, physically strengthen the cell wall and, might also play roles in plant-pathogen interactions $[14,15]$.

In general, both suberin and cutin contain fatty acids (FAs), $\omega$-hydroxy FAs and glycerol but suberin also contains high levels of $\alpha, \omega$-dicarboxylic acids, ferulic acid and fatty alcohols and its saturated aliphatics have longer chain lengths than in cutin (>C20 and $\mathrm{C} 16-18$, respectively) 
(Figure 1a) $[5,10]$. These composing monomers are linked essentially through acylglycerol or linear aliphatic ester bonds, building a three-dimensional network [16,17]. Fungal degradation of plant polyesters has been reported to involve the activity of carboxylesterases, namely cutinases [18-20]. Despite high complexity and redundancy, it is well established that FAs can be used by filamentous fungi via $\beta$-oxidation leading to the production of acetyl-CoA $[21,22]$. Downstream pathways, such as the glyoxylate and the tricarboxylic acid cycles and gluconeogenesis, enable the fungus to use FAs as sole carbon and energy sources.

In plants, suberin is ingrained between a primary and a tertiary cell wall and cross-linked to the other cell wall polymers, hence its extraction usually destroys its chemical skeleton [5]. This is still a major obstacle [5] and has restrained most biodegradation studies to the use of cutin films [23-25] and/or mixtures of nearly non-esterified suberin or cutin samples [26-28]. However, near-native suberin can be efficiently extracted from cork (outer bark of Quercus suber L.) through selective hydrolysis of acylglycerol ester bonds but leaving most linear aliphatic ester bonds intact [29-32] (Figure 1). In the present contribution we demonstrate that suberin macromolecules could be used as sole carbon source by Aspergillus nidulans. During fungal growth, synergetic action of cutinases, lipases and long chain fatty alcohol modifying enzymes

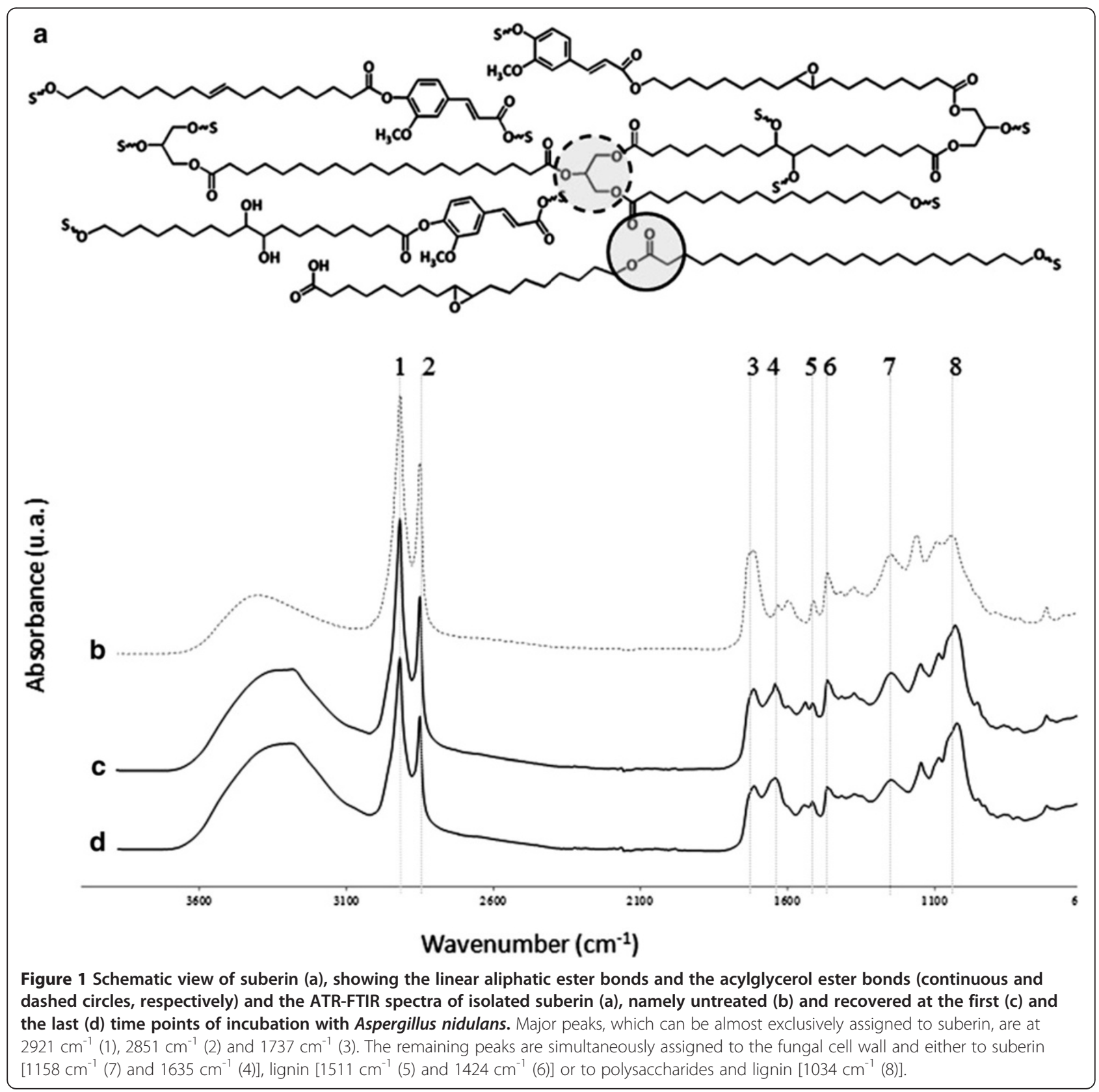


released suberin long chain fatty acids (LCFAs) that were metabolised via peroxisomal $\beta$-oxidation. Other major alterations included the onset of sexual development and the boost of secondary metabolism.

\section{Methods \\ Chemicals}

If not otherwise stated chemicals were of the highest analytical grade and purchased from Sigma Aldrich (USA). Suberin was extracted from cork using cholinium hexanoate as previous described [30], afterwards freeze dried and kept at $-20^{\circ} \mathrm{C}$ until used. The same process was used to recover the residual suberin upon fungal incubation. All suberin samples were analysed using attenuated total reflectance Fourier transform infrared spectroscopy (ATR-FTIR) as previously described $[30,32]$. The selected ATR-FTIR spectra should be regarded as representative (ten replicates).

\section{Culture conditions}

Fungal cultures with a density of $A$. nidulans (FGSC A4) conidia of $10^{5}$ per $\mathrm{mL}(5 \mathrm{~mL}$ six-well plates, minimal media [33] containing $1 \% \mathrm{w} / \mathrm{v}$ glucose) were grown for two days $\left(27^{\circ} \mathrm{C}\right.$, dark, $\left.90 \mathrm{rpm}\right)$ for the establishment of a mycelia mat against the bottom of the wells (control). Afterwards, old media were replaced by minimal media containing $0.1 \% \mathrm{w} / \mathrm{v}$ of suberin as sole carbon source, and cultures were grown, in the same conditions, for additional two, four, six or fifteen days. At each time point mycelia and the corresponding media filtrate were recovered and preserved at $-80^{\circ} \mathrm{C}$ [33]. Additional control cultures were prepared containing $0.1 \% \mathrm{w} / \mathrm{v}$ of $\mathrm{C} 16$ glyceryl tripalmitate or C8 octyl octanoate (contain acylglycerol ester and linear ester bonds, respectively) instead of suberin. Controls were processed as described above.

\section{Microscopic analyses}

Scanning Electron Microscopy (SEM) analysis of lyophilised fungal mycelia were performed using an analytical field emission gun scanning electron microscope (FEGSEM: JEOL $7001 \mathrm{~F}$ with Oxford light elements EDS detector) operated at 5-10 kV. After the LIVE/DEAD assay [34] (evaluates culture viability) and the safranin staining (detects the extracellular matrix typical of fungal biofilm [35]), mycelia were evaluated using a DM5500 B microscope (Leica) under fluorescent (49 DAPI and N21 filter sets) or white light, respectively. 10x and 63x magnification objectives were used, respectively and images were captured with a DFC420 C camera (Leica). In all tests the selected micrographs should be regarded as representative.

\section{Secretome analyses}

Extracellular protein was recovered from the culture filtrates using denaturing precipitation conditions [33]. $25 \mu \mathrm{g}$ of protein (bovine serum albumin equivalents accordingly to the Bradford protocol) were loaded in a precast gel (Criterion ${ }^{\mathrm{Tx}} \mathrm{XT}$ precast 1D gel $4-12 \%$ BisTris) and allow to migrate for $1 \mathrm{~cm}$. The gel was stained with Instant Blue (Gentaur BVBA), sliced into 5 bands; (each cut into 1-2 $\mathrm{mm}$ cubes), first reduced, alkylated and de-stained, then digested by trypsin (Promega). The peptides were first desalted and finally fractionated by reverse phase separation in an Ultimate 3000 NanoLC System coupled to a LTQ-OrbiTrap Elite MS that was operated in data-dependent mode, automatically switching between MS and MS2, using XCalibur software. Data was processed in Mascot using Proteome Discoverer by searching against the SwissProt Fungi (released on January $21^{\text {st }}$, 2013) and NCBI databases. Protein identification was done using a set of pre-defined filters and a minimum confidence of 95\%. Full details in Additional file 1. Glycerol in the culture media filtrates (40 times concentrated, duplicates) was analysed by chromatography as reported before [36]. Quantification detection limits were $0.01 \mathrm{~g} \cdot \mathrm{L}^{-1}$. The additional control cultures (see above) were also analysed.

\section{RNA isolation and CRNA preparation}

Total RNA was isolated from fungal mycelia (grounded to powder using mortar and pestle in liquid nitrogen) using the RNeasy Plant Mini Kit (QIAGEN) and further purified by ethanol precipitation [37]. Quantification and purity of RNA were determined on a NanoDrop 1000 Spectrophotometer (Thermo Scientific) and RNA integrity analysed using an Agilent 2100 Bioanalyser with a RNA 6000 Nano Assay (Agilent Technologies). Fragmented and biotinylated cRNA was obtained by following GeneChip 3' IVT Express Kit protocols. Briefly, 100 ng of total RNA were used for the synthesis of cDNA, which was further in vitro transcribed into labelled cRNA. After purification and fragmentation, the size distribution of the cRNA and fragmented cRNA were assessed in an Agilent 2100 Bioanalyzer with a RNA 6000 Nano Assay.

\section{Microarray processing}

The custom array FungiANC (Affymetrix) contains a total of 20,012 transcripts from the genetic information of A. nidulans and Neurospora crassa available at the Broad Institute database (http://www.broadinstitute.org) and is based on a Perfect Match-only (PM) design with $11 \mathrm{mi}-$ cron feature size. Each transcript is represented by 11 probes (25-mer each). See full details in Additional file 2. The array processing was performed accordingly to Affymetrix GeneChip protocols (biological triplicates). A total of $200 \mu \mathrm{l}$ of the hybridisation mixture containing $10 \mu \mathrm{g}$ of fragmented cRNA was hybridised on arrays for 16 hours at $45^{\circ} \mathrm{C}$. Standard post hybridisation washes and doublestain protocols (FS450_0001) were used on an Affymetrix GeneChip Fluidics Station 450, in conjunction with the GeneChip Hybridisation Wash and Stain Kit (Affymetrix). 
Arrays were scanned on an Affymetrix GeneChip Scanner 3000 7G. Array quality parameters were analysed by Expression Console Software (Affymetrix) for Robust Multiarray Averaging summarised data and confirmed to be in the recommended range. The data discussed in this publication have been deposited in NCBI's Gene Expression Omnibus [38] and are accessible through GEO Series accession number GSE54427 (http://www.ncbi.nlm.nih.gov/ geo/query/acc.cgi?acc=GSE54427).

\section{Microarray data analysis}

DNA-Chip Analyzer (dChip) 2010 (http://www.dchip.org) was used applying a probeset mask file considering only the A. nidulans probes (9675 transcripts). The normalised CEL intensities of the 12 arrays (Invariant Set Normalisation Method [39,40]), were used to obtain model-based gene expression indices based on a Perfect Match-only model $[39,40]$. dChip Log2 expression data were imported into R v2.13.0. Differentially expressed genes (analysis was carried out with Bioconductor LIMMA package, www.bioconductor.org), included only the probe sets with adjusted $p$-value $\leq 0.01$ and fold-change $(\mathrm{FC}) \geq 2$. See full details in Additional file 1 .

\section{Quantitative real-time PCR analysis ( $q$ RT-PCR)}

Based on the sequences of $A$. nidulans genes (Aspergillus Genome Database, http://www.aspergillusgenome.org/), all oligonucleotide pairs were designed using the GeneFisher2 web tool (http://bibiserv.techfak.uni-bielefeld.de/ genefisher2) and produced by Thermo Fisher Scientific (see Additional file 1 for the list of the oligonucleotides used in this study). The qRT-PCR analysis was performed in a CFX96 Thermal Cycler (Bio-Rad), using the SsoFast EvaGreen Supermix (Bio-Rad), $250 \mathrm{nM}$ of each oligonucleotide and the cDNA template equivalent to $1 \mathrm{ng}$ of total RNA, at a final volume of $10 \mu \mathrm{l}$ per well, in three technical and three biological replicates. The PCR conditions were: enzyme activation at $95^{\circ} \mathrm{C}$ for $30 \mathrm{~s}$; 40 cycles of denaturation at $95^{\circ} \mathrm{C}$ for $10 \mathrm{~s}$ and annealing/ extension at $59^{\circ} \mathrm{C}$ for $15 \mathrm{~s}$; and melting curve obtained from $65^{\circ} \mathrm{C}$ to $95^{\circ} \mathrm{C}$, consisting of $0.5^{\circ} \mathrm{C}$ increments for $5 \mathrm{~s}$. Data analyses were performed using the CFX Manager software (Bio-Rad). The expression of each gene was taken as the relative expression in pair-wise comparisons of consecutive time points. The expression of all target genes was normalised by the expression of the 60S ribosomal protein L33-A gene, AN2980, selected as internal control due to its constant levels in all time points.

\section{Functional annotation}

Annotations for all the genes represented on the FungiANC genome array were obtained from the Broad Institute database (www.broadinstitute.org) and the Aspergillus Genome Database (AspGD, www.aspgd.org). See full details in
Additional file 2. The differentially expressed genes for each biological condition were classified using the FungiFun web annotation tool [41] and the Functional Catalogue (FunCat). The significant hits ( $p$-value $\leq 0.05)$ were defined using the identities present on the chip as the background.

\section{Results \& discussion \\ Aspergillus nidulans transcriptome on suberin - enrich- ment analysis}

Pair-wise comparisons were used to identify differentially expressed genes (adjusted $p$-value $<0.01$ and $|\mathrm{FC}|>2$ ) between the control (grown on glucose) and during growth on suberin for two, four or six days (hereafter defined as first, mid and last time points, respectively) Additional file 2 . Within the differentially expressed genes (4198 constituting nearly half of the transcripts in the microarray), 32\% (1357 genes) can be specifically associated with the switch of the substrate (control $v s$ first time point) and were enriched in functional categories associated with the metabolism of alkanes, alkenes, alkanals and alkanols (MIPS 01.20.05.03) and the oxidation of fatty acids (MIPS 2.25), as well as cellular sensing and response to external stimulus (MIPS 33.11) and cell type differentiation (MIPS 43), among others (Figure 2b, Additional file 3). Pair-wise comparison of consecutive time points, henceforward systematically used, showed that among the enriched functional categories at the mid time point some were associated with increased nutrient starvation response (MIPS 32.01.11) and alterations in fatty acid metabolism (MIPS 01.06.05), along with major alterations in cell cycle (MIPS 10) and cell fate (MIPS 40) Additional file 3. In addition, those enriched at the last time point revealed e.g. an increased stress response (MIPS 32.01) and development of ascospores (MIPS 43.01.03.09). In particular, the degradation/modification of exogenous ester compounds (MIPS 32.10.09) can be associated with cleavage of ester bonds in suberin. The intensity of the major peak assigned to ester bonds (1737 $\mathrm{cm}^{-1}$ which can be exclusively assigned to the $\mathrm{C}=\mathrm{O}$ stretch of ester groups) in the ATR-FTIR spectra of suberin decreased significantly after fungal incubation (Figure 1b).

\section{Autolysis and primary metabolism}

Autolysis occurred after switching from glucose to suberin media. Major up-regulation of pepJ (AN7962, Table 1) and up-regulation of prtA (AN5558) resulted in accumulation of the encoded proteins in the secretome (Table 2). Both proteases play a role in the degradation of empty hyphae during starvation [42,43]. Out of the 25 protein species identified in the secretome, ten are involved in cell wall remodelling events typical of autolysis and show, in general, high consistency with the transcriptome data (Table 2). Several other cell wall remodelling genes related with autolysis were up-regulated along 

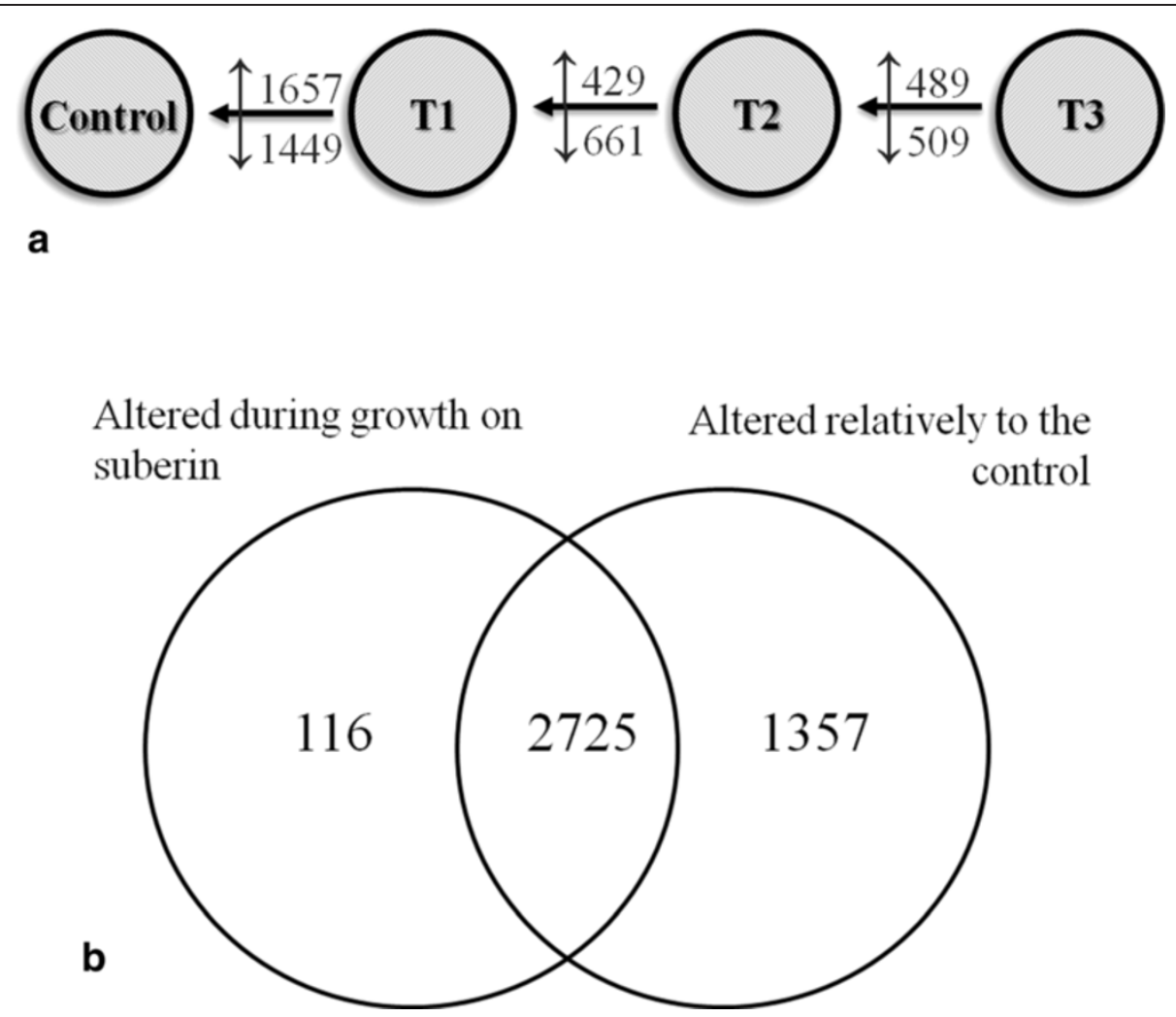

Figure 2 Number of differentially expressed genes during Aspergillus nidulans growth on suberin in pair-wise comparisons of consecutive times (a), discriminating the up- $(\uparrow)$ and the down- $(\downarrow)$ regulated genes, and Venn diagram highlighting the number of genes that were responsive only at the first time point on suberin when compared with the control (b).

cultivation: AN9042, mutA (AN7349), AN7613, AN2690 and AN8392, chiB (AN4871) [44,45], engA (AN0472) [46] and nagA (AN1502) [44] (Table 1 and Additional file 2). Glucuronan lyase A gene (AN0012) has been reported to be up-regulated in $A$. nidulans during starvation [47] but here its expression level at the first time point was exceedingly higher (Table 1). The encoded protein specifically breaks $\beta$-1,4-glucuronans, which are rarely found in the cell walls of Aspergilli [48] and absent in those of cork [6]. Hence, the functional role of AN0012 remains largely unclear. Few genes related with apoptosis (AN7500 and AN5712) $[49,50]$ or autophagy (AN2876 and AN5174) [51] were responsive along the incubation, suggesting that they played only a minor role during the fungus growth on suberin Additional file 2. As typically reported in A. nidulans after imposition of severe carbon starvation conditions, several putative major facilitator superfamily (MFS) transporter genes (AN9232, AN8084, AN6778, AN8502) [47,52] underwent major up-regulation (Table 1). The last two genes belong to uncharacterised secondary metabolite clusters [53], thus probably coding for specific transmembrane transporters. Some other MFS genes (AN5860, mstE and AN4180) and one auxin efflux carrier superfamily gene (AN8018) were amongst those showing major down-regulation (Table 1). $m s t E$ is induced in the presence of several repressing carbon sources and is dependent on the function of CreA (AN6195) [54]. Upregulation of $c r e A$, the carbon catabolite repressor A [55], at the first time point (Table 3 ) might be explained by a self-repression mechanism [56,57]. Gluconeogenesis activation might have occurred as suggested by the upregulation of the pathway transcription activator (acuM, AN6293) and of phosphoenolpyruvate carboxykinase gene (acuF, AN1918) which, together with the fructose 1,6bisphosphatase gene (acuG, AN5604, not differentially expressed in the suberin media), regulate this pathway [58] (Table 4, Additional file 2). The 4-aminobutyrate (GABA) shunt is inactive during gluconeogenesis [59], likely coordinated with major down-regulation of putative glutamate decarboxylase gene (AN7278) at the first time point (Table 1). Major up-regulation of the fermentation transcription activator alcR (AN8978, Table 1) at the last time point occurred but the aldehyde dehydrogenase gene (AN0554) [60] was not differentially expressed Additional file 2.

The transcriptional regulation of the assimilatory nitrate system in A. nidulans differs in high availability or limiting glucose conditions [61]. AreA is the major nitrogen 
Table 1 List of the differentially expressed genes showing the highest fold changes (FCs) in pair-wise comparison of consecutive time points during Aspergillus nidulans growth on suberin

\begin{tabular}{|c|c|c|c|c|c|c|c|c|}
\hline \multicolumn{3}{|c|}{$\begin{array}{l}\text { First time point of suberin incubation (between the } \\
\text { control and second day of incubation on suberin) }\end{array}$} & \multicolumn{3}{|c|}{$\begin{array}{l}\text { Mid time point of suberin incubation (between the } \\
\text { second and fourth days of incubation on suberin) }\end{array}$} & \multicolumn{3}{|c|}{$\begin{array}{l}\text { Last time point of suberin incubation (between } \\
\text { the fourth and sixth days of incubation on suberin) }\end{array}$} \\
\hline FC & Gene \# & Description & FC & Gene \# & Description & FC & Gene \# & Description \\
\hline \multicolumn{9}{|c|}{ Up-regulated } \\
\hline 358.7 & AN6000 & Asperthecin polyketide synthase (aptA) & 31.8 & AN7369 & GMC oxidoreductase & 23.5 & AN9230 & $\begin{array}{l}\text { Monooxygenase associated } \\
\text { with secondary metabolism }\end{array}$ \\
\hline 305.8 & AN5309 & Putative cutinase 1 (cut 1 ) & 22.0 & AN10030 & Putative alkaline serine protease & 9.6 & AN6778 & MFS transporter \\
\hline 278.8 & AN7962 & Metalloproteinase (pep) & 22.7 & AN9224 & Monooxygenase & 8.6 & AN10026 & Oxidoreductase \\
\hline 252.5 & AN9042 & Mutanase & 21.7 & AN9493 & Putative acetyltransferase (ngn12) & 7.0 & AN11202 & $\begin{array}{l}\text { Putative DMATS-type } \\
\text { aromatic prenyltransferase }\end{array}$ \\
\hline 107.4 & AN0012 & Glucuronan lyase A & 20.1 & AN9227 & $\begin{array}{l}\text { Dioxygenase associated } \\
\text { with secondary metabolism }\end{array}$ & 6.6 & AN8392 & Melibiase subfamily \\
\hline 91.6 & AN7812 & $\begin{array}{l}\text { Putative sterigmatocystin } \\
\text { biosynthesis protein (stcN) }\end{array}$ & 19.4 & AN5348 & Fatty acyl-CoA reductase & 6.6 & AN8106 & $\begin{array}{l}\text { Dioxygenase associated } \\
\text { with secondary metabolism }\end{array}$ \\
\hline 90.1 & AN7349 & Mutanase (mutA) & 18.7 & AN7613 & Putative chitinase & 6.4 & AN8473 & RNA polimerase II transcription factor \\
\hline 58.5 & AN2623 & $\begin{array}{l}\text { Acyl-CoA:6-aminopenicillanic- } \\
\text { acid-acyltransferase (aatA) }\end{array}$ & & & & 5.8 & AN8978 & Regulatory protein (alcR) \\
\hline 46.7 & AN11013 & $\begin{array}{l}\text { Probable sterigmatocystin biosynthesis } \\
\text { P450 monooxygenase (stcL) }\end{array}$ & 16.4 & AN2690 & Putative $\beta$-1,3-glucanase & 5.5 & AN8084 & MFS transporter \\
\hline 35.0 & AN3931 & $\begin{array}{l}\text { Meiotic expression up-regulated } \\
\text { protein } 14 \text { (pilB) }\end{array}$ & & & & 5.0 & AN8520 & $\begin{array}{l}\text { protein required for terrequinone } \\
\text { A biosynthesis (tdiE) }\end{array}$ \\
\hline 33.4 & AN6835 & NADPH-cytochrome P450 reductase & 16.2 & AN9232 & MFS transporter & 5.0 & AN3369 & $\mathrm{Zn}_{2}-\mathrm{Cys}_{6}$ transcription factor (clrB) \\
\hline 32.8 & AN7811 & $\begin{array}{l}\text { Putative sterigmatocystin } \\
\text { biosynthesis protein (stcO) }\end{array}$ & 16.6 & AN9234 & $\begin{array}{l}\text { Oxidoreductase associated } \\
\text { with secondary metabolism }\end{array}$ & 4.9 & AN6747 & $\mathrm{C} 2 \mathrm{H} 2$ type zinc finger transcription factor \\
\hline \multicolumn{2}{|c|}{$33<\mathrm{FC}<87$} & $\begin{array}{l}\text { Predicted proteins and poorly } \\
\text { characterised genes }\end{array}$ & \multicolumn{2}{|c|}{$17<\mathrm{FC}<147$} & $\begin{array}{l}\text { Predicted proteins and poorly } \\
\text { characterised genes }\end{array}$ & \multicolumn{2}{|c|}{$4<\mathrm{FC}<8$} & $\begin{array}{l}\text { Predicted proteins and } \\
\text { poorly characterised genes }\end{array}$ \\
\hline \multicolumn{3}{|c|}{$\begin{array}{c}\text { AN1532, AN8995, AN6476, AN9301, AN7235, AN2330, } \\
\text { AN4825, AN2913, AN0461, AN8656, AN1666, } \\
\text { AN0488, AN1952 }\end{array}$} & \multicolumn{3}{|c|}{$\begin{array}{l}\text { AN6020, AN4970, AN7958, AN7957, AN5292, AN5319, AN8037, AN1155, } \\
\text { AN2400, AN2710, AN7655, AN8162, AN9235, AN11163, AN1946 }\end{array}$} & \multicolumn{3}{|c|}{$\begin{array}{l}\text { AN7419, AN7092, AN2779, AN3881, AN7091, AN7395, } \\
\text { AN8955, AN2658, AN2859, AN1719, AN2376, AN5422 }\end{array}$} \\
\hline \multicolumn{9}{|c|}{ Down-regulated } \\
\hline-39.5 & AN0399 & Nitrate transporter (nrtB) & -18.6 & AN7824 & $\begin{array}{l}\text { Probable sterigmatocystin biosynthesis } \\
\text { P450 monooxygenase (stcB) }\end{array}$ & -13.1 & AN2583 & Glyceraldehyde 3-phosphate dehydrogenase \\
\hline-35.9 & AN1008 & Nitrate transporter $(\mathrm{crnA})$ & -18.1 & AN8356 & Alcohol dehydrogenase & -9.8 & AN1666 & Nucleolar GTP-binding protein 2 \\
\hline-35.4 & AN1006 & Nitrate reductase (niaD) & -14.1 & AN7804 & $\begin{array}{l}\text { Putative sterigmatocystin } \\
\text { biosynthesis monooxygenase (stcW) }\end{array}$ & -8.6 & AN2861 & Putative F-box protein \\
\hline-26.1 & AN7539 & Hydrophobin & -12.6 & AN7818 & $\begin{array}{l}\text { Probable sterigmatocystin biosynthesis } \\
\text { P450 monooxygenase (stcF) }\end{array}$ & -6.3 & AN5228 & NADH:flavin oxidoreductase/NADH oxidase \\
\hline-24.4 & AN7392 & Choline transporter Hnm1 & -11.8 & AN7807 & $\begin{array}{l}\text { Putative sterigmatocystin biosynthesis } \\
\text { protein (stcT) }\end{array}$ & & & \\
\hline
\end{tabular}


Table 1 List of the differentially expressed genes showing the highest fold changes (FCs) in pair-wise comparison of consecutive time points during Aspergillus nidulans growth on suberin (Continued)

\begin{tabular}{|c|c|c|c|c|c|c|c|c|}
\hline & & & -11.5 & AN7815 & Fatty acid synthase subunit a & -5.6 & AN4180 & MFS transporter \\
\hline & & & -11.4 & AN5860 & MFS monosaccharide transporter (mstE) & & & \\
\hline \multirow[t]{2}{*}{-24.1} & \multirow[t]{2}{*}{ AN2859 } & \multirow[t]{2}{*}{ Dihydrodipicolinate synthetase } & -11.2 & AN7825 & $\begin{array}{l}\text { Putative sterigmatocystin biosynthesis } \\
\text { polyketide synthase (stcA) }\end{array}$ & \multirow[t]{2}{*}{-5.6} & \multirow[t]{2}{*}{ AN10619 } & \multirow[t]{2}{*}{ Glutamate decarboxylase } \\
\hline & & & -11.2 & AN4135 & Stearoyl-CoA desaturase (sdeB) & & & \\
\hline-23.9 & AN4131 & $\mathrm{Na}^{+} / \mathrm{H}^{+}$antiporter & -11.1 & AN7814 & Fatty acid synthase subunit $\beta$ & \multirow[t]{2}{*}{-5.5} & \multirow[t]{2}{*}{ AN7169 } & \multirow[t]{2}{*}{ Flavohemoprotein (fhbA) } \\
\hline-23.8 & AN7278 & Glutamate decarboxylase & -10.8 & AN7806 & Versicolorin reductase & & & \\
\hline-21.7 & AN4119 & MFS multidrug transporter & -10.6 & AN3763 & Siderochrome-iron transporter & \multirow[t]{2}{*}{-5.5} & \multirow[t]{2}{*}{ AN3264 } & \multirow[t]{2}{*}{ MFS multidrug transporter } \\
\hline-19.0 & AN3776 & MFS transporter & -10.0 & AN7811 & $\begin{array}{l}\text { Putative sterigmatocystin biosynthesis } \\
\text { protein }(s t c O)\end{array}$ & & & \\
\hline \multicolumn{2}{|c|}{$-61<\mathrm{FC}<-18$} & $\begin{array}{l}\text { Predicted proteins and poorly } \\
\text { characterised genes }\end{array}$ & \multicolumn{2}{|c|}{$-28<\mathrm{FC}<-10$} & Predicted protein and poorly characterised genes & -16 & $<F C<-5$ & $\begin{array}{l}\text { Predicted protein and poorly } \\
\text { characterised genes }\end{array}$ \\
\hline \multicolumn{3}{|c|}{$\begin{array}{l}\text { AN2595, AN7214, AN5305, AN8081, AN6946, AN4108, AN7378, } \\
\text { AN9220, AN6128, AN8981, AN3333, AN6930, AN4128, AN6932, } \\
\text { AN8670 }\end{array}$} & \multicolumn{3}{|c|}{$\begin{array}{l}\text { AN7397, AN3251, AN8544, AN6661, AN7817, AN8314, } \\
\text { AN2722, AN0169, AN7812, AN7809, AN7915, AN11013 }\end{array}$} & \multicolumn{3}{|c|}{$\begin{array}{l}\text { AN2571, AN9310, AN7949, AN5489, AN0421, AN0461, } \\
\text { AN9102, AN9378, AN7960, AN3305, AN0728, AN0247, } \\
\text { AN0288, AN8974, AN0838, AN8544, AN3314 }\end{array}$} \\
\hline
\end{tabular}


Table 2 List of the extracellular protein species identified in the secretome of Aspergillus nidulans at the last time point of growth on suberin

\begin{tabular}{|c|c|c|c|c|c|c|c|c|c|c|c|}
\hline \multirow{2}{*}{$\begin{array}{l}\text { Gene } \\
\text { number }\end{array}$} & \multirow[t]{2}{*}{ Description } & \multirow{2}{*}{$\begin{array}{c}\mathrm{MW} \\
{[\mathrm{kDa}]}\end{array}$} & \multirow[t]{2}{*}{ calc. pl } & \multirow[t]{2}{*}{ SignalPa } & \multicolumn{2}{|c|}{ SwissProt } & \multicolumn{2}{|c|}{ NCBI } & \multicolumn{3}{|c|}{ Microarray data* } \\
\hline & & & & & $\begin{array}{l}\text { Total } \\
\text { peptides }\end{array}$ & $\overline{\operatorname{Cov}(\%)}$ & $\begin{array}{l}\text { Total } \\
\text { peptides }\end{array}$ & $\operatorname{Cov}(\%)$ & First $^{c}$ & Mid $^{c}$ & Last $^{\mathrm{c}}$ \\
\hline \multicolumn{12}{|c|}{ Plant Polymer Degrading Enzymes } \\
\hline AN5309 & Cutinase (Cut1) & 22.4 & 7.3 & Y & 3 & 12.21 & 2 & 9.09 & 305.8 & -3 & 1.5 \\
\hline AN8046 & Triacylglycerol lipase & 31.3 & 4.6 & Y & $-\mathrm{b}$ & $-\mathrm{b}$ & 7 & 47.28 & 10.4 & -1.6 & 2.7 \\
\hline AN3613 & $\beta-1,4-x y l a n a s e(X \ln A)$ & 24.1 & 6.3 & Y & 4 & 36 & 4 & 36 & 6.5 & -2.2 & -1.1 \\
\hline AN7401 & $\beta$-1,4-endoxylanase (XInE) & 37.7 & 5.5 & Y & $-\mathrm{b}$ & $-\mathrm{b}$ & 3 & 11.83 & -1.3 & -1.4 & 1 \\
\hline AN1818 & $\beta$-1,4-xylanase $C(X \ln C)$ & 35.4 & 5.2 & Y & 5 & 20.49 & $-b$ & $-b$ & 1.2 & -1.2 & -1.3 \\
\hline AN8477 & $\beta$-1,4-xylosidase & 60.3 & 5.4 & $\mathrm{~N}$ & $-b$ & $-b^{b}$ & 4 & 9.71 & 1.6 & 1.1 & 1.1 \\
\hline AN2828 & $\beta$-glucosidase L (BglL) & 77.8 & 4.8 & Y & 14 & 21.71 & 12 & 22.52 & -1.2 & -1.3 & 2.4 \\
\hline \multicolumn{12}{|c|}{ Fungal Development } \\
\hline AN0472 & $\beta$-1,3-endoglucanase A (EngA) & 97.8 & 5.7 & Y & $-\mathrm{b}$ & $-\mathrm{b}$ & 6 & 8.49 & 5.8 & 1.1 & 1.2 \\
\hline AN7950 & $\beta$-1,3-endoglucosidase (EglC) & 46.7 & 4.6 & Y & 10 & 28.39 & 10 & 28.39 & $-d$ & $-d$ & $-d^{d}$ \\
\hline AN4825 & $\beta$-1,3-glucosidase & 96.5 & 5.5 & Y & $-\mathrm{b}$ & $-\mathrm{b}$ & 11 & 17 & 42.5 & 1.1 & 1.2 \\
\hline AN2395 & $\beta$-glucuronidase & 68.5 & 4.8 & Y & $-b$ & $-\mathrm{b}$ & 14 & 28.06 & 7.4 & -1.4 & 1.2 \\
\hline AN4871 & Chitinase B (ChiB) & 44.2 & 5.6 & Y & $-\mathrm{b}$ & $-\mathrm{b}$ & 34 & 80.65 & 12.7 & 1.4 & 1.1 \\
\hline AN2017 & a-glucosidase (AgdA) & 109.6 & 5.2 & Y & $-\mathrm{b}$ & $-\mathrm{b}$ & 6 & 8.17 & -1.5 & -1.9 & 2.3 \\
\hline AN8445 & Aminopeptidase $Y$ & 53.7 & 6.8 & Y & $-\mathrm{b}$ & $-\mathrm{b}$ & 6 & 19.68 & 1.8 & -1.5 & 1.6 \\
\hline AN2366 & Serine protease & 25.4 & 4.4 & Y & $-b$ & $-\mathrm{b}$ & 3 & 17.27 & $-d$ & $-d^{d}$ & $-d^{d}$ \\
\hline AN5558 & Alkaline serine protease (PrtA) & 42.2 & 5.3 & Y & 8 & 32.75 & 7 & 32.75 & 5.4 & 1.3 & 1.1 \\
\hline AN7962 & Metalloproteinase (PepJ) & 37.4 & 5.1 & $\mathrm{~N}$ & 6 & 18.36 & 6 & 18.36 & 278.8 & 1.8 & 1.2 \\
\hline AN4245 & Ceramidase & 80 & 5 & Y & $-\mathrm{b}$ & $-\mathrm{b}$ & 7 & 14.8 & 1.6 & 1.5 & 1.8 \\
\hline AN9339 & Catalase B (CatB) & 79.1 & 5.1 & Y & 23 & 35.6 & 20 & 34.54 & -2.4 & 1.8 & 1 \\
\hline \multicolumn{12}{|c|}{ Miscellaneous } \\
\hline AN3351 & Uncharacterised & 63.2 & 5.1 & Y & $-\mathrm{b}$ & $-\mathrm{b}$ & 5 & 12.91 & 1.6 & -1.4 & -1.1 \\
\hline AN3246 & Uncharacterised & 22.3 & 6.4 & $\mathrm{~N}$ & $-b$ & $-\mathrm{b}$ & 4 & 29.8 & 2 & -1.3 & 2 \\
\hline AN6273 & Allergenic Asp F13 & 16.3 & 4.8 & Y & $-\mathrm{b}$ & $-\mathrm{b}$ & 3 & 32.91 & -1 & -1.5 & -1.4 \\
\hline AN5879 & $\begin{array}{l}\text { Phosphatidylglycerol/phosphatidylinositol } \\
\text { transfer protein }\end{array}$ & 18.3 & 5 & Y & 9 & 53.25 & 9 & 53.25 & 1 & 1 & -1 \\
\hline AN8979 & Alcohol dehydrogenase I (AlcA) & 36.9 & 6 & $\mathrm{~N}$ & 5 & 25.57 & 5 & 25.57 & -15.1 & -8.8 & 1 \\
\hline AN8043 & Uncharacterised & 16.9 & 4.8 & $\mathrm{~N}$ & $-\mathrm{b}$ & $-{ }_{-}^{b}$ & 3 & 26.42 & -4 & -2 & -1 \\
\hline
\end{tabular}

${ }^{a}$ SignalP was used to predict secretion signals $[67,68]$ and ${ }^{b}$ not found in the database search. Corresponding microarray data are shown for comparison. ${ }^{*}$ Values highlighted in bold have $|F C|>2$ and p-value $<0.01$ in the microarray data; ${ }^{c}$ Fold changes (FCs) in pair-wise comparison of consecutive time points; ${ }^{d}$ not represented in the chip.

regulatory protein, however, under glucose limiting conditions, the nitrogen status-sensing regulator AreB controls the expression of the nitrate catabolic genes [61,62]. Major down-regulation of nitrate reductase niaD (AN1006) and of nitrate transporters (AN1008, AN0399) (Table 1) was consistent with the down-regulation of nitrite reductase niiA (AN1007) and the up-regulation of areB (AN6221) Additional file 2. At the last time point, the inducible nitric oxide-detoxifying flavohaemoglobin gene (AN7169, fhbA) showed major down-regulation (Table 1). This gene is coregulated with niaD and niiA, yet its expression is AreAindependent $[63,64]$.

\section{Development and secondary metabolism}

During growth on suberin the mycelia mat surrounded the water-insoluble substrate, disrupting the fungal biofilm formed in the control, i.e. loss of hyphae alignment and disruption of the extracellular polysaccharide matrix (Figure 3). The expression levels of some $A$. nidulans orthologs of $A$. fumigatus genes coding in important pathways of biofilm formation were consistent with the loss of biofilm morphology [65], including major down-regulation of hydrophobin gene (AN7539) (Table 1).

Sexual development in $A$. nidulans was induced during growth on suberin, similar to that observed before on 
Table 3 qRT-PCR analysis of a selected set of genes coding putative lipid hydrolysing enzymes or major regulatory proteins

\begin{tabular}{|c|c|c|c|c|c|c|c|c|}
\hline \multirow[t]{2}{*}{ Carbon source } & \multirow[t]{2}{*}{ Gene } & \multirow[t]{2}{*}{ Encoded protein } & \multicolumn{3}{|c|}{$q \mathrm{RT}-\mathrm{PCR}$} & \multicolumn{3}{|c|}{ Microarray* } \\
\hline & & & First $^{d}$ & Mid $^{d}$ & Last $^{d}$ & First $^{d}$ & $\mathrm{Mid}^{\mathrm{d}}$ & Last $^{\mathrm{d}}$ \\
\hline \multirow[t]{12}{*}{ Suberin } & AN6195 & CreA & 8.3 & -2.3 & -1.9 & 4.3 & -1.4 & -1.6 \\
\hline & AN1052 & VeA & 2.5 & -1.0 & -1.0 & 2.6 & -1.3 & 1.0 \\
\hline & AN7050 & FarA & 2.3 & -1.7 & 1.3 & 2.8 & -1.7 & 1.3 \\
\hline & AN5309 & Cut1 & 119.9 & -3.1 & 1.8 & 305.8 & -3.0 & 1.5 \\
\hline & AN7541 & Cut2 & -1.5 & -1.0 & 1.5 & -1.3 & -1.1 & 1.1 \\
\hline & AN7180 & Cutinase & 1.2 & 1.1 & 1.9 & 1.3 & -1.2 & -1.1 \\
\hline & AN5267 & FaeC & 3.2 & -2.1 & 1.7 & 3.0 & -2.4 & 1.1 \\
\hline & AN2697 & Putative lipase ${ }^{a}$ & -1.8 & 1.4 & 1.8 & 1.6 & -1.7 & -1.1 \\
\hline & AN5777 & Putative lipase & 2.9 & 1.0 & 1.7 & 2.9 & -1.2 & 1.6 \\
\hline & AN8046 & Putative lipase & 4.1 & -1.1 & 2.3 & 10.4 & -1.6 & 2.7 \\
\hline & AN8900 & Putative lipase $^{b}$ & -1.8 & 1.7 & 1.5 & 1.6 & 1.6 & -1.1 \\
\hline & AN4748 & Uncharacterised protein ${ }^{c}$ & 31.8 & -1.8 & -3.8 & 24.6 & -1.7 & -4.5 \\
\hline \multirow[t]{4}{*}{ Glyceryl tripalmitate } & AN5309 & Cut1 & 1.4 & 36.4 & -7.8 & & & \\
\hline & AN7541 & Cut2 & 2.7 & -3.8 & -1.3 & & & \\
\hline & AN7050 & FarA & 3.7 & -1.7 & -1.7 & & & \\
\hline & AN8046 & Putative lipase & 8.2 & 2.3 & -19.4 & & & \\
\hline \multirow[t]{4}{*}{ Octyl octanoate } & AN5309 & Cut1 & 3.3 & 14.0 & -1.6 & & & \\
\hline & AN7541 & Cut2 & 4.1 & -1.2 & -3.0 & & & \\
\hline & AN7050 & FarA & 2.7 & -1.3 & -1.4 & & & \\
\hline & AN8046 & Putative lipase & 2.8 & 5.8 & -11.7 & & & \\
\hline
\end{tabular}

Values represent the relative expression of selected genes in pair-wise comparisons of consecutive time points. The expression of each gene was normalised by the expression of the 60S ribosomal protein L33-A gene (AN2980).

*Values highlighted in bold have $|\mathrm{FC}|>2$ and $p$-value $<0.01$ in the microarray data; ${ }^{\text {a }}$ contains feruloyl esterase and tannase domains, high homology with feruloyl esterase B in N. crassa; ${ }^{b}$ orthologue of A. niger An09g02270, which encodes a triacylglycerol lipase; 'orthologue of S. cerevisiae NOP6, which is necessary for rRNA-binding protein required for $40 \mathrm{~S}$ ribosomal subunit biogenesis. ${ }^{\mathrm{d}}$ Fold changes (FCs) in pair-wise comparison of consecutive time points. Corresponding microarray data are shown for comparison.

FAs [22]: Hülle cells and few ascospores were detected at the first and last time points, respectively; whereas cleistothecia and numerous ascospores were observed only after fifteen days of cultivation (Figure 4). The breach of cleistothecia might explain the few ascospores detected at the last time point. In agreement, mutA (mutanase associated with the use of glucose reserves during the formation of Hülle cells [66]) and AN10030 (alkaline serine protease involved in the biosynthesis of the ascopore cell wall $[67,68]$ ) underwent major upregulation at first and mid time points, respectively. In addition, MAT1 (AN2755) and MAT2 (AN4734) genes, which encode the transcription factors considered the master switchers of sexual development in A. nidulans $[69,70]$, were up-regulated at the first time point Additional file 2 . The expression profile of the vast majority of genes associated with sexual development in A. nidulans, including steA (AN2290), was consistent with the onset of sexual development [71]. The few exceptions included genes signalling response to nitrogen/carbon limitation [61], namely down- and up-regulation of sexual development activators (csnB, AN4783 and noxA, AN5457) and repressors (silG,
AN0709; $c p c A$, AN3675 and rosA, AN5170), respectively Additional file 2.

The expression of genes regulating fungal growth and development is known to be coordinated with those coding in the biosynthesis of asperthecin, penicillin and sterigmatocystin [72]. Moreover, exogenous addition of mannans to the growth media (oligosaccharides that might be released during autolysis) increases penicillin production in Penicillium sp. [73]. In fact, genes in asperthecin (AN6000, aptA), penicillin (AN2623) and sterigmatocystin (AN7812, AN11013 and AN7811) clusters were amongst those more profoundly affected at the first time point (Table 1). Analyses of the expression levels of the other in-cluster genes confirmed these findings, e.g. up-regulation of genes in sterigmatocystin cluster included the aflR regulator (AN7820) [74], the polyketide synthase (AN7825) and the fatty acid synthase genes (AN7814 and AN7815) Additional file 2. At the mid time point, sterigmatocystin biosynthesis probably decreased, since genes encoding the synthase (AN7825) and some auxiliary enzymes (AN7804, AN7806, AN7811, AN7818 and AN7824) become major down- 
Table 4 List of Aspergillus nidulans differentially expressed genes (pair-wise comparison of consecutive time points), putatively involved in suberin degradation (Continued)

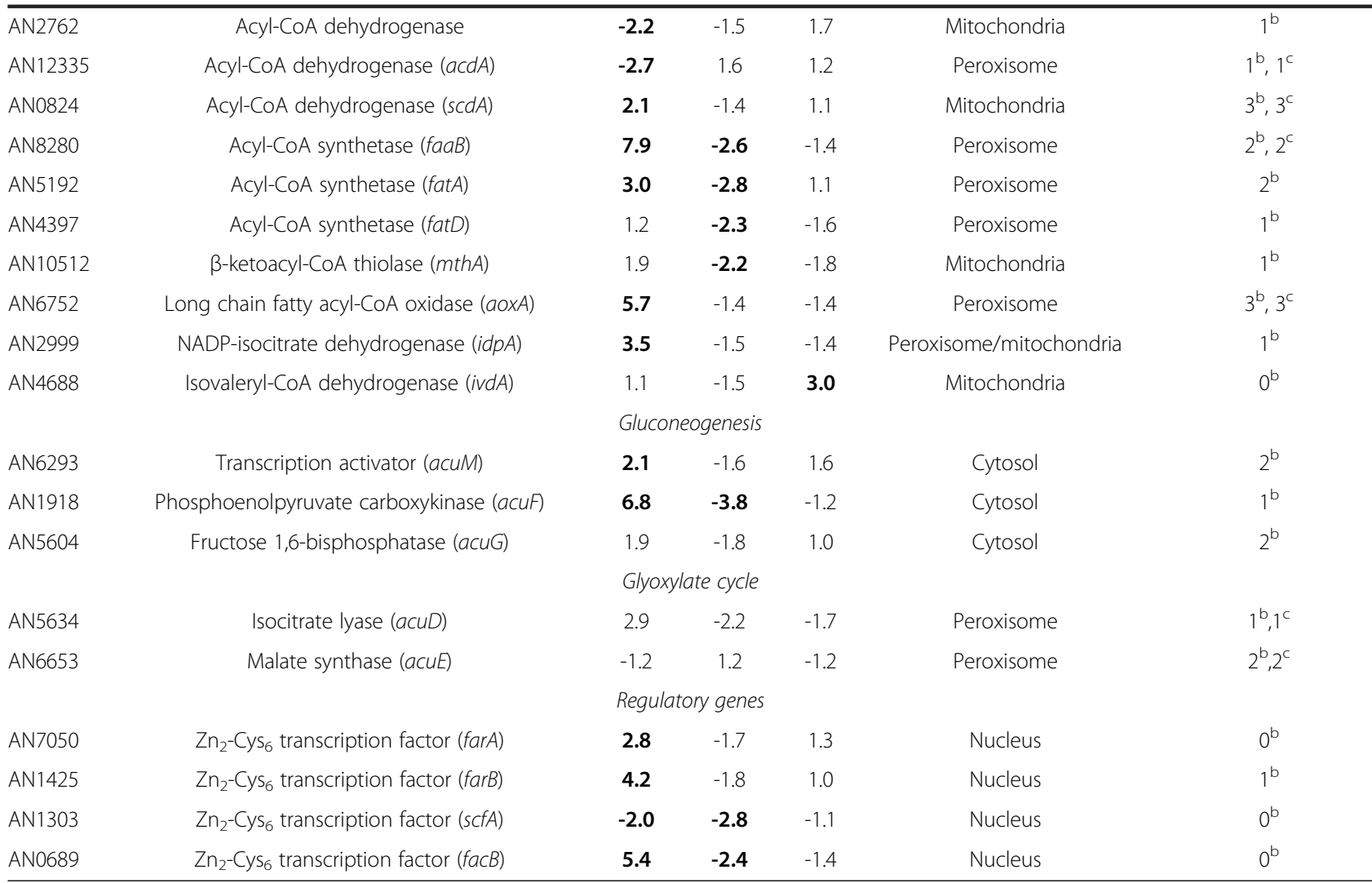

*values highlighted in bold have $|\mathrm{FC}|>2$ and $p$-value $<0.01$ in the microarray data; ${ }^{\text {a) }}$ Sub-cellular location was attained using Pedant Database (http://pedant.gsf.de). ${ }^{b}$ the number of predicted binding sites for farA was manually searched according to the conserved sequence 5 '-CCTCGG or its reverse complement sequence (5'-CCGAGG) within $1 \mathrm{~Kb}$ of the upstream region of the start codon of listed genes; ${ }^{C}$ number of predicted binding sites as previous reported [22]. ${ }^{\mathrm{d}}$ Fold changes (FCs) in pair-wise comparison of consecutive time points.

regulated (Table 1). Suberin also induced major alterations in numerous genes coding in uncharacterised clusters (AN9234, AN9227, AN9230, AN11202, AN8106 and AN8520). Overall, 65 secondary metabolite gene clusters, out of the 71 estimated by now in this fungus [53], were responsive but only in a few clusters the synthase gene was largely affected. Supplementation of the growth media with suberoylanilide hydroxamic acid induced several secondary metabolite synthases in A. niger [75] and potato suberin augmented the diversity of the secondary metabolites biosynthesised by Streptomyces sp. [76].

Twelve genes involved in the control of development and carbon metabolism, as well as in suberin degradation, were selected to validate the microarray data by $q$ RT-PCR (Table 3). The tested genes included four genes which putatively encode lipid hydrolysing enzymes (AN7541, AN7180, AN2697 and AN8900) that showed FC $\leq 2$ in the microarray. All the analysed genes, with the exception of AN2697, showed a profile of expression along the cultivation similar to that of the microarray data.

\section{Degradation of suberin: lipid hydrolysis}

Several genes encoding polyester hydrolysing enzymes were induced during the fungus growth on suberin (Table 4). Data made apparent that Cut1 played the major role in suberin initial degradation: the encoding gene (AN5309) was extensively up-regulated (Table 1) and the enzyme was present in the secretome (Table 2). Cut1 hydrolyses aliphatic polyesters [77] and potato suberin $[18,19,26]$. FarA and FarB are major transcription activators of genes involved in FAs utilisation [22]. Deletion of farA (AN7050) eliminates induction of a number of genes by both small chain fatty acids (SCFAs) and LCFAs, while deletion of $\operatorname{farB}$ (AN1425) eliminates SCFAs induction [22]. As expected, farA and farB were both up-regulated at the first time point (Table 4). The down-regulation of scfA on suberin (Table 4, AN1303, which encodes a similar transcription factor and its deletion leads to farB deleted mutant phenotype [22]) might be explained by its repression under nitrogen limiting conditions [64]. With the exception of cut1, the expression 


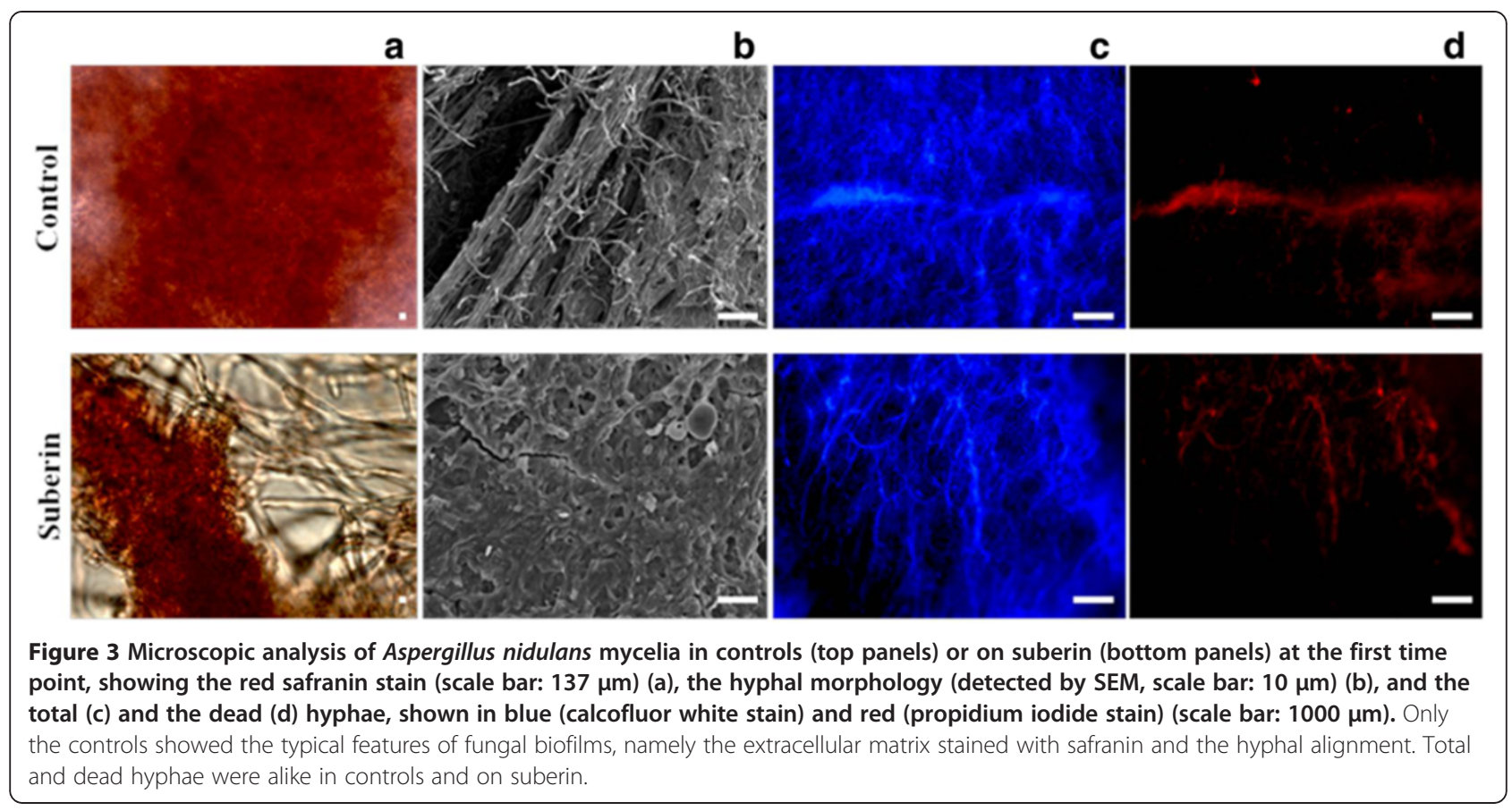

levels of the other three cutinase genes were kept at basal levels (AN10346) or underwent minor alterations (cut2 AN7541 and AN7180) (Tables 3 and 4). FarA regulation of $A$. nidulans cutinase genes might be similar to that reported in Fusarium solani [26]. In the pathogenic fungus, cut 1 is strongly induced by cutin monomers under the regulation of CTF1 $\alpha$ - which has $70 \%$ similarity with FarA and the same binding motif, CCTCGG - but cut2 and cut 3 are expressed at basal levels. Similar regulation was noticed in media containing octyl octanoate or glyceryl tripalmitate as sole carbon sources (Table 3). In the suberin media major up-regulation of cut 1 occurred at an earlier time point probably because it contains $c a$. 4 wt\% of soluble "cutin-like" monomers [30,78]. Glycerol could not be detected in the suberin culture filtrates likely because 80$90 \%$ of the acylglycerol bonds in suberin were hydrolysed during its extraction (Figure 1a) [31]. In agreement, glycerol catabolic genes [79], namely glycerol kinase (AN5589, glcA) and FAD-dependent glycerol 3-phosphate dehydrogenase (AN1396) were not differentially expressed. When glyceryl tripalmitate was used as sole carbon source, glycerol was detected, namely $0.063 \pm 0.003$ and $0.0201 \pm 0.0018 \mathrm{~g} \cdot \mathrm{L}^{-1}$ at the first and the last time point, respectively.

Only Cut1 and AN8046, putative lipid hydrolysing enzymes, could be detected in the secretome (Table 2). Gene expression data corroborate the hypothesis that far $A$ regulates cut1 and AN8046 during A. nidulans utilisation of suberin (Table 4), as well as octyl octanoate or glyceryl tripalmitate (Table 3) as sole carbon sources. The farA ortholog gene of $A$. oryzae regulates not only cutL (cutinase gene) but also the putative lipid hydrolysing enzyme genes $m d l B$ (ortholog of AN8046) and tglA (70\% homology with AN10346) [80]. It also regulates hsbA (ortholog of AN6795) that encodes hydrophobic surface binding protein probably involved in the recruitment of CutL to the FAs surface. AN6795 was also stimulated by suberin (Table 4). In addition, during Fusarium oxysporum growth on wheat oil, the transcription factor ctf1 regulates both cut1 and lip1 [81], which is amongst the lipase genes more strongly induced [82] (n.b. Lip1 shows high homology to AN8046 protein).

Several other putative lipase genes, namely AN2602, AN6464, AN5777, AN4573, AN1799, AN6773, in addition to AN2697 and AN8900 (FC < 2, Table 3) were also stimulated by suberin (Table 4). Their regulation was variable, except AN4573 (increased along the incubation) and AN6773 (increased at first and mid time points). The latter, as well as AN1799 and AN5321, have been associated with unresolved secondary metabolite gene clusters in A. nidulans [53] and likely are not involved in suberin degradation.

Eleven genes encoding $\mathrm{ABC}$ transporters carrying transmembrane domains were up-regulated at the first time point. $\mathrm{ABC}$ transporters are generally assumed to be involved in multidrug resistance, yet more recent studies have shown their physiological significance e.g. in oxidative stress response, pathogenicity and excretion of siderophore peptide breakdown products [83]. A role in penicillin secretion was proposed for the A. nidulans AtrD transporter, which belongs to the subfamily ABC-C [84]. atrD (AN2300) up-regulation at the first time point agrees with major up-regulation of penicillin synthase gene 


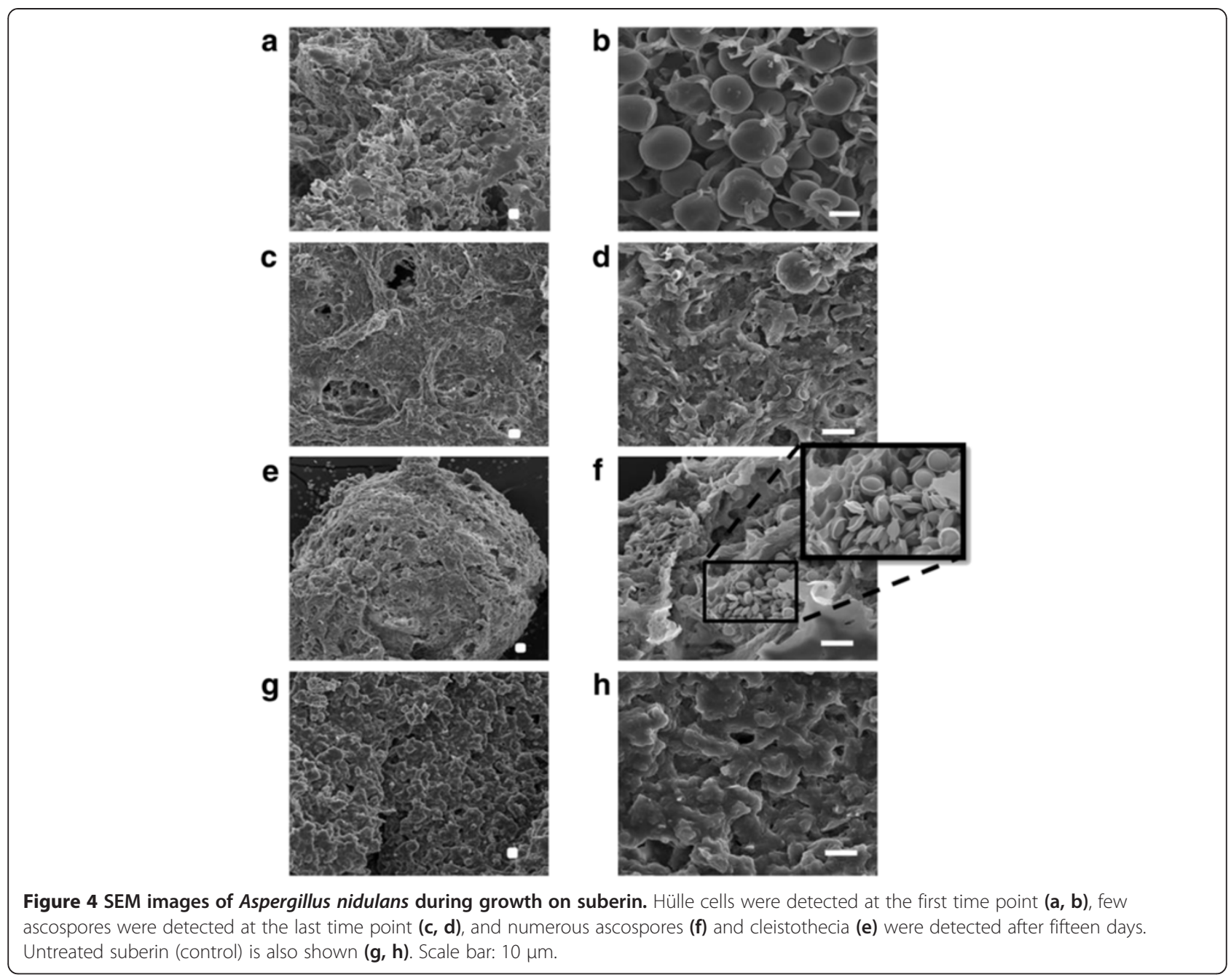

(Table 1). Up-regulation of numerous ABC-G transporter genes (AN6581, AN8813, AN6369, AN0771, AN8892 and AN8489) might imply a possible involvement in the transmembrane transport of suberin hydrolysed monomers. The function of this subfamily of transporters remains largely unknown with putative roles in e.g. excretion of hydrophobic and/or lipid molecules [85]. In addition, suberin possibly stimulated the formation of eisosomes playing a role in the endocytosis of lipid cargos [86], as suggested by the major up-regulation of pilB (AN3931) (Table 1).

Hydrolysed suberin LC fatty alcohols (Figure 1a) need further modification before entering FAs $\beta$-oxidation pathways. $\omega$-Hydroxy fatty acid oxidation was probably mediated by NADPH-cytochrome $\mathrm{P} 450$ reductase fusion enzyme (AN6835), as well as by LC fatty alcohol oxidase (AN0623). Both encoding genes were up-regulated during growth on suberin (Tables 1 and 4). The first enzyme might catalyse the oxidation of the LC fatty alcohols to carboxylic acids and of mid-chain saturation functionalities to vic-diols $[87,88]$. LC fatty alcohol oxidases have been shown to catalyse the oxidation of $\omega$-hydroxy fatty alcohols in Candida cloacae [89] and the encoding genes display usually a peroxisomal targeting sequence, notwithstanding their cellular localisation in A. nidulans remains unknown. Due to different substrate specificities and/or cellular compartmentalisation, possibly AN6835 and AN0623 enzymes have acted sequentially during A. nidulans growth on suberin. The hypothesis that LC fatty alcohols undergo modification by LC fatty alcohol oxidase in the peroxisome merits further analysis.

\section{Degradation of suberin: $\beta$-oxidation}

Current understanding of FAs utilisation in A. nidulans indicates significant complexity and redundancy in $\beta$-oxidation pathways $[21,22,90]$. FAs are activated by FA-CoA synthetases to their acyl-CoA derivates, which are processed by FA-CoA oxidases or dehydrogenases. Each round of $\beta$-oxidation produces a chain-shortened fatty-acyl-CoA (which undergoes further rounds) and an acetyl-CoA, which is channelled into the tricarboxylic acid or glyoxylate cycles. In addition, $\beta$-oxidation substrates are 
actively transported across the mitochondria and/or the peroxisome membrane by carnitine shuttles, ADP/ATP carriers or $A B C$ transporter proteins. Suberin stimulated two peroxisomal FA-CoA synthetase genes, namely $f a a B$ (AN8280) and fatA (AN5192) (Table 4) but none of the well characterised mitochondrial ones (e.g. facA (AN5626) and pcsA (AN5833), Additional file 2). It seems that hydrolysed suberin monomers were essentially processed via peroxisomal $\beta$-oxidation pathways (Table 4 ) and that, as previously suggested, FaaB is the major peroxisomal FA-CoA synthetase, while the remaining ones (FatA-D (AN5192, AN5877, AN6649, AN4397), FaaA (AN6114) and AN4659) display high functional redundancy [91]. Activation of peroxisomal $\beta$-oxidation agrees with the upregulation of antA, a peroxisomal ATP carrier (AN0257) and of $a c u J$, a mitochondrial/peroxisomal carnitine acetyltransferase (AN6279) (Table 4). Uncharacterised transporters for the peroxisomal translocation of activated LCFAs have been suggested [22]; justifying the basal expression levels of pxa1 and pxa2 (AN10078 and AN1014, $\mathrm{ABC}$ transporter proteins).

Suberin stimulated the expression of $a \circ x A$ (AN6752) but not $a o x B$ (AN6765), both encoding peroxisomal fatty-acylCoA oxidases (Table 4) but AoxA plays the major role during growth on LCFAs [92]. Deletion of aoxA leads to growth impairment on LCFAs but not on SCFAs or very long chain fatty acids (VLCFAs) [21]. aoxA stimulation is consistent with the observation that suberin hydrolysis releases three times more LCFAs than VLCFAs [30,78]. Only three peroxisomal fatty-acyl-CoA dehydrogenase genes (out of seven) were responsive at the first time point, namely acdA (AN12335), AN1699 and AN7320 genes (Table 4). Deletion of either acdA or AN7320 has not led to any growth impairment on FAs, suggesting that the encoded enzymes display high redundancy [92]. Botrytis cinerea BC1G_13535 gene, which displays 78\% of homology with AN1699, was amongst the highest up-regulated genes coding in FAs $\beta$-oxidation during Lactuca sativa infection [93]. The hypothesis that this fatty-acyl-CoA dehydrogenase plays a major role in $A$. nidulans degradation of plant FAs calls for its functional characterisation.

LCFAs $\beta$-oxidation is shuttled between the peroxisomes and the mitochondria, typically when the produced chainshortened fatty-acyl-CoA is a butyryl-CoA (C4) [92]. At the first time point, most mitochondrial fatty-acyl-CoA dehydrogenase genes were down-regulated (AN2762, AN6761 and AN9162), except scdA (AN0824) [22] that was up-regulated. The remaining mitochondrial $\beta$-oxidation genes, in general, decreased at the mid and/or last time points, including the well characterised $m t h A$, a $\beta$-ketoacyl-CoA thiolase gene (AN10512), as well as $a c u H$, a mitochondrial carnitine/acyl-carnitine carrier (AN5356) (Table 4). The only exception was $i v d A$, an isovaleryl-CoA dehydrogenase gene (AN4688) that was up-regulated, together with $m c c B$ (AN4687), at the last time point (Table 4). This might imply that the fungus started using leucine as a catabolic source; both genes are clustered with $m c c A$ (AN4690) in the leucine catabolic pathway [94].

The glyoxylate bypass is absolutely required for growth on carbon sources that produce acetyl-CoA and is dependent on isocitrate lyase (AcuD) and malate synthase (AcuE) activities. Transcription of acuD (AN5634) and $a c u E$ (AN6653) is regulated by FA and acetate induction via the FacB activator, but $f a c B$ (AN0689) mutations do not prevent growth on FAs [22]. Up-regulation of farA and $\operatorname{farB}$ (FA-induced) and of $f a c B$ (acetate-induced) led to up-regulation of $a c u D$ but not of $a c u E$ (Table 4). Previous studies have demonstrated that the imposition of several stresses might lead to both fluctuating mRNA and irregular protein expression levels in A. nidulans [95].

\section{Degradation of phenolic suberin}

Release of ferulic acid during fungal growth on potato suberin has been suggested to involve the activity of feruloyl esterases (Fae) [5], notwithstanding a direct proof is still lacking. Ferulic acid release probably justifies the up-regulation of faeC (AN5267) (Table 3). Ferulic acid degradation might involve the activity of 2,3-dihydroxybenzoate carboxylyase [96], of which the encoding gene $d h b D$ (AN6723) was up-regulated at the first time point (Additional file 2). Several downstream products have been reported in different fungal strains, however the associated enzymes remain largely unknown [96]. Nevertheless, final degradation of the formed aromatics might involve the homogentisate, benzoate or the salicylate pathways [97], and several genes coding in these pathways were found to be up-regulated (e.g. AN10950, hmgA (AN1897), $h p d A$ (AN1899), maiA (AN1895), Additional file 2) in the suberin media.

\section{Degradation of other cell wall constituents}

Several polysaccharides degrading enzymes were identified in the A. nidulans secretome on suberin at the last time point (Table 2, Additional file 4), in general, agreeing with the transcriptome data (Additional file 2). They included the $\beta$-glucosidase BglL (AN2828) and the xylanase XlnA (AN3613), of which the encoding genes were found up-regulated at the last and the first time points, respectively. The other $\beta$-glucosidase genes were upregulated at the first (bglM, AN7396) and mid time points (eglB (AN5214) and AN3046) and could not be detected in the secretome. $x \ln R$ (AN7610), which encodes the xylanolytic/cellulolytic transcriptional activator, was up-regulated at the last time point (consistent with creA decrease [98]), notwithstanding the upregulation of the $x \ln A$ and $x \ln B$ (AN9365) at the earlier time points. The additional polysaccharide degrading enzymes detected in the secretome were not consistent 
with the transcriptome data (Table 2), including XlnC (AN1818) and XlnE (AN7401). Probably the upregulation of their corresponding genes occurred at an intermediate time point. The poor correlation between transcriptomic and proteomic data has been previously reported [95,99], often related to mechanisms associated with mRNA turnover and/or efficiency of translation [100] or to transcription on demand of certain mRNA pools [101], among other reasons, including experimental noise [102]. Protein species grouped in the miscellaneous category (six, of which half have a predicted intracellular localisation) might be associated with cell lysis, as highlighted by the presence of alcohol dehydrogenase I (alcA, AN8979) (Table 2).

\section{Conclusions}

Previous studies on A. nidulans colonisation of cork revealed that suberin remained unaltered [103] probably because the outermost lignin-enriched cell wall layers hampered its degradation. Here we have shown that
A. nidulans was able to utilise suberin macromolecules as sole carbon source (Figure 1b) and that the fungus also underwent sexual development (Figure 4) and boosted secondary metabolism (Table 1 ). We propose the suberin degradation and utilisation pathway in A. nidulans, as depicted schematically in Figure 5 . Amongst the up-regulated genes encoding lipid hydrolysing enzymes only two were detected in the secretome, namely Cut1 and AN8046 (Tables 2, 3 and 4). In particular, out of the four cutinase genes, only cut 1 expression pattern was correlated to that of farA, similar to that described in plant pathogenic fungi $[22,81]$. $\omega$-Hydroxy fatty acid oxidation reactions (mediated by either NADPH-cytochrome P450 reductase or LC fatty alcohol oxidase, Tables 1 and 4), are likely involved in the modification of suberin LC fatty alcohols, even if their cellular compartmentalisation remains uncertain. The hydrolysed suberin monomers were essentially composed of LCFAs, hence activated to their corresponding acyl-CoA derivatives probably by FaaB; the major peroxisomal fatty acyl-CoA synthetase in this

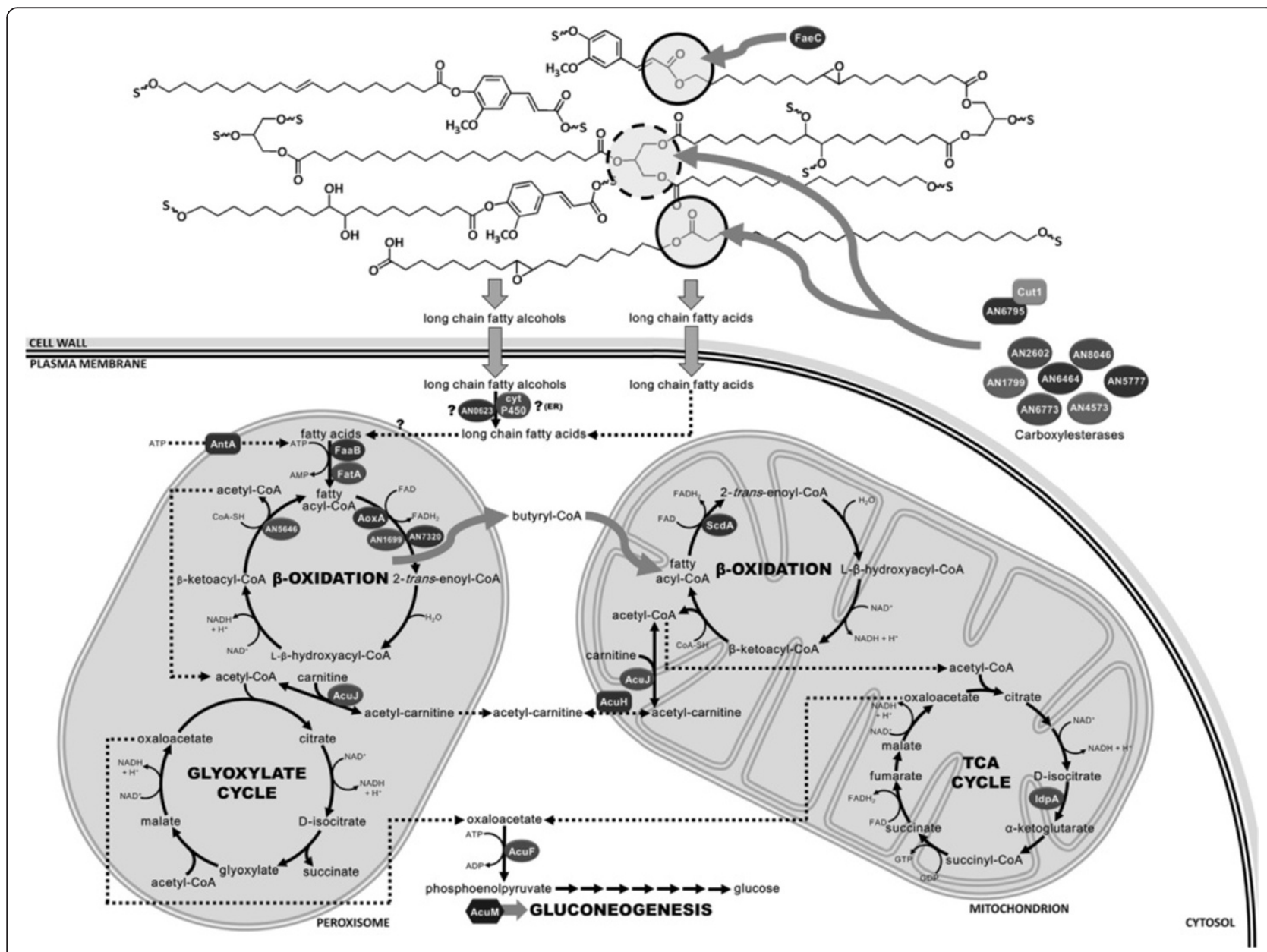

Figure 5 Schematic view of putative suberin degradation and utilisation pathways in Aspergillus nidulans. For sake of clarity, some steps and intermediates are omitted and only the proteins of which the encoding genes were up-regulated in the first and the mid time points are represented. Uncertainties in the cellular compartmentalisation or activity of the enzyme are indicated by question marks. 
fungus [92]. Despite high functional redundancy of additional peroxisomal FA-CoA synthetase genes, fat $A$ showed the highest up-regulation on suberin. Upregulation of $a \circ x A$ also occurred, agreeing with the idea that the encoded fatty-acyl-CoA oxidase plays a major role during growth on LCFAs [21]. In addition, among the peroxisomal fatty-acyl-CoA dehydrogenase genes upregulated here, AN1699 underwent the highest upregulation (Table 4), similar to that reported for its $B$. cinerea ortholog during plant infection [93]. The core binding sequence for FarA, typically overrepresented in the promoter region of genes required for growth on FAs, is not present in all related genes up-regulated during $A$. nidulans growth on suberin (Table 3). Some unknown regulatory elements are certainly yet to be discovered, further emphasised by the down-regulation of some lipid hydrolysing genes carrying the FarA recognition site.

\section{Availability of supporting data}

The data sets supporting the results of this article are included within the article (and its additional files).

\section{Additional files}

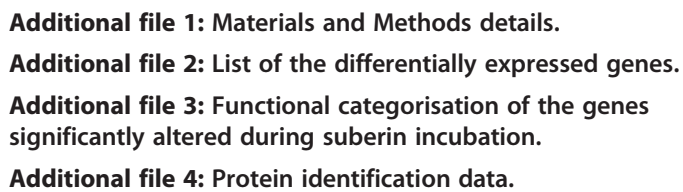

\section{Abbreviations}

FC: Fold change; FAs: Fatty acids; SCFAs: Small chain fatty acids; LCFAs: Long chain fatty acids; VLCFAs: Very long chain fatty acids; ATR-FTIR: Attenuated total reflectance Fourier transform infrared spectroscopy; SEM: Scanning electron microscopy; qRT-PCR: Quantitative real-time PCR analysis; MIPS: Munich Information Center for Protein Sequences.

\section{Competing interests}

The authors declare that they have no competing interests.

\section{Authors' contributions}

CSP supervised the project and the interpretation of data and prepared the final version of the manuscript. All authors have done substantial contributions to the acquisition, analysis and interpretation of data, and contributed to the drafting of the manuscript: IM (experimental set up, transcriptomics and secretome analyses and preparation of the initial draft of the manuscript), DOH (PCR analyses), CM (microscopy studies), PCA, JH and JDB (set up of the custom microarray), HG and RF (suberin isolation and spectroscopy), HG and JDB (statistics), JR and CL (secretome analyses). All authors read and approved the final version of the manuscript.

\section{Authors' information}

This research constitutes an important milestone of C. Silva Pereira's team (established in 2008) that is addressing a major scientific question: How fungi perceive and interact with the environment? What makes our research approach distinctive is the merging of fundamental biology research with chemical expertise. Such multidisciplinary environment has inspired the development of a new extraction method for suberin - a structural component of the plant cell wall. Elucidating how fungi utilise and degrade plant polyesters strongly impacts on our understanding of plant-fungi interactions and nutrients cycling. Complementary to this research, we have reported that the plant polyester can be reconstituted as a material that combines bactericidal and antibiofouling properties. We are particularly interested in further elucidating plant polyesters physiological roles and to promote the clinical use of plant polyester based materials.

\section{Acknowledgements}

The work was partially supported by a grant from Iceland, Liechtenstein and Norway through the EEA financial mechanism (Project PT015), and FCT through the grants PEst-OE/EQB/LA0004/2013, PTDC/QUI-QUI/120982/2010 and PTDC/AAC-CLI/119100/2010

IM, DOH, PCA and RF are grateful to Fundação para a Ciência e a Tecnologia (FCT), Portugal, for the fellowships SFRH/BD/38378/2007, SFRH/BD/66396/ 2009, SFRH/BD/66030/2009, SFRH/BD/48286/2008, respectively and HG to Fundação Calouste Gulbenkian, Portugal for the fellowship 21-95587-B. We are thankful to Dr. Tiago Martins (ITQB), who read and critically commented the final manuscript.

\section{Author details}

'Instituto de Tecnologia Química e Biológica, Universidade Nova de Lisboa, Av. da República, 2780-157 Oeiras, Portugal. ${ }^{2}$ Instituto de Biologia Experimental e Tecnológica (iBET), Av. da República, 2781-901 Oeiras, Portugal. ${ }^{3}$ Proteomics Platform, Centre de Recherche Public - Gabriel Lippmann, Belvaux, Luxembourg. ${ }^{4}$ Cancer Genomics Research Laboratory, Division of Cancer Epidemiology and Genetics, National Cancer Institute, $\mathrm{NIH}$, DHHS, 8717 Grovemont Circle, 20877 Gaithersburg, MD, USA (previously, the Scientific Computing department, Samuel Roberts Noble Foundation, USA). ${ }^{5}$ Instituto Gulbenkian de Ciência, Rua da Quinta Grande 6, 2780-156 Oeiras, Portugal.

Received: 18 March 2014 Accepted: 16 July 2014 Published: 21 July 2014

\section{References}

1. Heredia A, Matas A, Dominguez E: Investigating plant lipid biopolymers. Biochem Educ 2000, 28(1):50-51.

2. Winkler A, Haumaier L, Zech W: Insoluble alkyl carbon components in soils derive mainly from cutin and suberin. Org Geochem 2005, 36(4):519-529.

3. Hamer U, Rumpel C, Dignac MF: Cutin and suberin biomarkers as tracers for the turnover of shoot and root derived organic matter along a chronosequence of Ecuadorian pasture soils. Eur J Soil Sci 2012, 63(6):808-819.

4. Harms H, Schlosser D, Wick LY: Untapped potential: exploiting fungi in bioremediation of hazardous chemicals. Nat Rev Microbiol 2011, 9(3):177-192.

5. Kolattukudy PE, Scheper T, Babel W, Steinbüchel A: Polyesters in higher plants. In Biopolyesters, Volume 71; 2001:1-49.

6. Pereira H: Cork: Biology, Production and Uses. Amsterdam: Elsevier; 2007.

7. van Fleet DS: Histochemistry and function of the endodermis. Bot Rev 1961, 27(2):165-220.

8. Yeats TH, Rose JKC: The formation and function of plant cuticles. Plant Physiol 2013, 163(1):5-20.

9. Schreiber L: Transport barriers made of cutin, suberin and associated waxes. Trends Plant Sci 2010, 15(10):546-553.

10. Pollard M, Beisson F, Li YH, Ohlrogge JB: Building lipid barriers: biosynthesis of cutin and suberin. Trends Plant Sci 2008, 13(5):236-246.

11. Javelle $M$, Vernoud V, Rogowsky PM, Ingram GC: Epidermis: the formation and functions of a fundamental plant tissue. New Phytol 2011, 189(1):17-39.

12. Kolattukudy PE: Biopolyester membranes of plants: cutin and suberin. Science 1980, 208(4447):990-1000.

13. Lulai EC, Corsini DL: Differential deposition of suberin phenolic and aliphatic domains and their roles in resistance to infection during potato tuber (Solanum tuberosum L.) wound-healing. Physiol Mol Plant Pathol 1998, 53(4):209-222.

14. Ranathunge $\mathrm{K}$, Schreiber $L$, Franke R: Suberin research in the genomics era: new interest for an old polymer. Plant Sci 2011, 180(3):399-413.

15. Garcia H, Ferreira R, Martins C, Sousa AF, Freire CSR, Silvestre AJD, Kunz W, Rebelo LPN, Silva Pereira C: Ex situ reconstitution of the plant biopolyester suberin as a film. Biomacromolecules 2014, 15(5):1806-1813.

16. Graça J, Schreiber L, Rodrigues J, Pereira H: Glycerol and glyceryl esters of w-hydroxyacids in cutins. Phytochemistry 2002, 61(2):205-215.

17. Graça J, Lamosa P: Linear and branched poly ( $\omega$-hydroxyacid) esters in plant cutins. J Agr Food Chem 2010, 58(17):9666-9674. 
18. Fernando G, Zimmermann W, Kolattukudy PE: Suberin-grown Fusarium solani f. sp pisi generates a cutinase-like esterase which depolymerizes the aliphatic components of suberin. Physiol Mol Plant Pathol 1984, 24(2):143-155.

19. Kontkanen $\mathrm{H}$, Westerholm-Parvinen A, Saloheimo M, Bailey M, Rättö M Mattila I, Mohsina M, Kalkkinen N, Nakari-Setälä T, Buchert J: Nove Coprinopsis cinerea polyesterase that hydrolyzes cutin and suberin. Appl Environ Microb 2009, 75(7):2148-2157.

20. Garcia Lepe R, Nuero OM, Reyes F, Santamaria F: Lipases in autolysed cultures of filamentous fungi. Lett Appl Microbiol 1997, 25(2):127-130.

21. Maggio-Hall LA, Keller NP: Mitochondrial $\beta$-oxidation in Aspergillus nidulans. Mol Microbiol 2004, 54(5):1173-1185.

22. Hynes MJ, Murray SL, Duncan A, Khew GS, Davis MA: Regulatory genes controlling fatty acid catabolism and peroxisomal functions in the filamentous fungus Aspergillus nidulans. Eukaryot Cell 2006, 5(5):794-805

23. Purdy R, Kolattukudy P: Hydrolysis of plant cuticle by plant pathogens. Properties of cutinase I, cutinase II, and a nonspecific esterase isolated from Fusarium solani pisi. Biochemistry 1975, 14(13):2832-2840.

24. Fan C-Y, Köller W: Diversity of cutinases from plant pathogenic fungi: diferential and sequential expression of cutinolytic esterases by Alternaria brassicicola. FEMS Microbiol Lett 1998, 158(1):33-38.

25. Bonnen AM, Hammerschmidt R: Cutinolytic enzymes from Colletotrichum lagenarium. Physiol Mol Plant Pathol 1989, 35(6):463-474

26. Li D, Sirakova T, Rogers L, Ettinger WF, Kolattukudy PE: Regulation of constitutively expressed and induced cutinase genes by different zinc finger transcription factors in Fusarium solani f. sp. pisi (Nectria haematococca). J Biol Chem 2002, 277:7905-7912.

27. Castro-Ochoa D, Peña-Montes C, González-Canto A, Alva-Gasca A, Esquivel-Bautista R, Navarro-Ocaña A, Farrés A: ANCUT2, an extracellular cutinase from Aspergillus nidulans induced by olive oil. Appl Biochem Biotechnol 2012, 166:1275-1290.

28. Yang $S, X u H$, Yan Q, Liu Y, Zhou P, Jiang Z: A low molecular mass cutinase of Thielavia terrestris efficiently hydrolyzes poly(esters). J Ind Microbiol Biotechnol 2013, 40:217-226.

29. Garcia H, Ferreira R, Petkovic M, Ferguson JL, Leitão $M C$, Gunaratne $H Q$, Seddon K, Rebelo L, Silva Pereira C: Dissolution of cork biopolymers in biocompatible ionic liquids. Green Chem 2010, 12:367-369.

30. Ferreira R, Garcia H, Sousa AF, Freire CSR, Silvestre AJD, Rebelo LPN, Silva Pereira C: Isolation of suberin from birch outer bark and cork using ionic liquids: a new source of macromonomers. Ind Crop Prod 2013, 44:520-527

31. Ferreira R, Garcia $H$, Sousa AF, Guerreiro M, Duarte FJS, Freire CSR, Calhorda MJ, Silvestre AJD, Rebelo LPN, Silva Pereira C: Unravelling the dual role of cholinium hexanoate ionic liquid as solvent and catalyst in suberin depolymerization. RSC Adv 2014, 4(1):2993-3002.

32. Ferreira R, Garcia H, Sousa AF, Petkovic M, Lamosa P, Freire CSR, Silvestre AJD, Rebelo LPN, Silva Pereira C: Suberin isolation from cork using ionic liquids: characterisation of ensuing products. New J Chem 2012, 36(10):2014-2024.

33. Carvalho MB, Martins I, Medeiros J, Tavares S, Planchon S, Renaut J, Nuñez O, Gallart-Ayala H, Galceran MT, Hursthouse A, Silva Pereira C: The response of Mucor plumbeus to pentachlorophenol: a toxicoproteomics study. J Proteomics 2013, 78:159-171.

34. Petkovic M, Hartmann DO, Adamová G, Seddon KR, Rebelo LPN, Silva Pereira C: Unravelling the mechanism of toxicity of alkyltributylphosphonium chlorides in Aspergillus nidulans conidia. New J Chem 2012, 36(1):56-63.

35. Seidler M, Salvenmoser S, Müller FM: In vitro effects of micafungin against Candida biofilms on polystyrene and central venous catheter sections. Int J Antimicrob Agents 2006, 28(6):568-573.

36. Carvalho MB, Martins I, Leitão MC, Garcia H, Rodrigues C, Romão VS, McLellan I, Hursthouse A, Silva Pereira C: Screening pentachlorophenol degradation ability by environmental fungal strains belonging to the phyla Ascomycota and Zygomycota. J Ind Microbiol Biotechnol 2009, 36(10):1249-1256.

37. Hartmann DO, Silva Pereira C: A molecular analysis of the toxicity of alkyltributylphosphonium chlorides in Aspergillus nidulans. New J Chem 2013, 37(5):1569-1577.

38. Edgar R, Domrachev M, Lash AE: Gene expression omnibus: NCBI gene expression and hybridization array data repository. Nucleic Acids Res 2002, 30(1):207-210
39. Li C, Wong WH: Model-based analysis of oligonucleotide arrays: expression index computation and outlier detection. Proc Natl Acad Sci U S A 2001, 98(1):31-36.

40. Li C, Wong WH: Model-based analysis of oligonucleotide arrays: model validation, design issues and standard error application. Genome Biol 2001, 2(8):1-11.

41. Priebe $S$, Linde J, Albrecht D, Guthke R, Brakhage AA: FungiFun: a web-based application for functional categorization of fungal genes and proteins. Fungal Genet Biol 2011, 48(4):353-358.

42. Emri T, Szzlágyi M, László K, M-Hamvas M, Pócsi I: PepJ is a new extracellular proteinase of Aspergillus nidulans. Folia Microbiol 2009, 54(2):105-109.

43. Szilágyi M, Kwon NJ, Bakti F, M-Hamvas M, Jámbrik K, Park H, Pócsi I, Yu JH, Emri T: Extracellular proteinase formation in carbon starving Aspergillus nidulans cultures - physiological function and regulation. J Basic Microbiol 2011, 51(6):625-634.

44. Pusztahelyi T, Molnár Z, Emri T, Klement É, Miskei M, Kerékgyártó J, Balla J, Pócsi l: Comparative studies of differential expression of chitinolytic enzymes encoded by chiA, chiB, chiC and nagA genes in Aspergillus nidulans. Folia Microbiol 2006, 51(6):547-554.

45. Shin KS, Kwon NJ, Kim YH, Park HS, Kwon GS, Yu JH: Differential roles of the ChiB chitinase in autolysis and cell death of Aspergillus nidulans. Eukaryot Cell 2009, 8(5):738-746.

46. Szilágyi $M$, Kwon NJ, Dorogi C, Pócsi I, Yu JH, Emri T: The extracellular $\beta-1,3$-endoglucanase EngA is involved in autolysis of Aspergillus nidulans. J Appl Microbiol 2010, 109(5):1498-1508.

47. Szilágyi M, Miskei M, Karányi Z, Lenkey B, Pócsi I, Emri T: Transcriptome changes initiated by carbon starvation in Aspergillus nidulans. Microbiol (UK) 2013, 159:176-190.

48. Muzzarelli RAA, Miliani M, Cartolari M, Tarsi R, Tosi G, Muzzarelli C: Polyuronans obtained by regiospecific oxidation of polysaccharides from Aspergillus niger, Trichoderma reesei and Saprolegnia sp. Carbohyd Polym 2000, 43(1):55-61.

49. Tsiatsiani L, Van Breusegem F, Gallois P, Zavialov A, Lam E, Bozhkov PV Metacaspases. Cell Death Differ 2011, 18(8):1279-1288.

50. Uren AG, O'Rourke K, Aravind L, Pisabarro MT, Seshagiri S, Koonin EV, Dixit VM Identification of paracaspases and metacaspases: two ancient families of caspase-like proteins, one of which plays a key role in MALT lymphoma. Mol Cell 2000, 6(4):961-967.

51. Savoldi M, Malavazi I, Soriani FM, Capellaro JL, Kitamoto K, da Silva Ferreira ME, Goldman MHS, Goldman GH: Farnesol induces the transcriptional accumulation of the Aspergillus nidulans Apoptosis Inducing Factor (AIF) like mitochondrial oxidoreductase. Mol Microbiol 2008, 70(1):44-59

52. Ren $\mathrm{Q}$, Chen K, Paulsen IT: TransportDB: a comprehensive database resource for cytoplasmic membrane transport systems and outer membrane channels. Nucleic Acids Res 2007, 35:D274-D279.

53. Inglis DO, Binkley J, Skrzypek MS, Arnaud MB, Cerqueira GC, Shah P, Wymore F, Wortman JR, Sherlock G: Comprehensive annotation of secondary metabolite biosynthetic genes and gene clusters of Aspergillus nidulans, $A$. fumigatus, A. niger and A. oryzae. BMC Microbio/ 2013, 13:91.

54. Forment JV, Flipphi M, Ramón D, Ventura L, MacCabe AP: Identification of the $m s t E$ gene encoding a glucose inducible, low affinity glucose transporter in Aspergillus nidulans. J Biol Chem 2006, 281(13):8339-8346.

55. Dowzer CEA, Kelly JM: Analysis of the CreA gene, a regulator of carbon catabolite repression in Aspergillus nidulans. Mol Cell Biol 1991, 11(11):5701-5709.

56. David H, Krogh AM, Roca C, Akesson M, Nielsen J: CreA influences the metabolic fluxes of Aspergillus nidulans during growth on glucose and xylose. Microbiol (UK) 2005, 151:2209-2221.

57. Strauss J, Horvath HK, Abdallah BM, Kindermann J, Mach RL, Kubicek CP The function of CreA, the carbon catabolite repressor of Aspergillus nidulans, is regulated at the transcriptional and post-transcriptional level. Mol Microbiol 1999, 32(1):169-178.

58. Hynes MJ, Szewczyk E, Murray SL, Suzuki Y, Davis MA, Lewis HMS: Transcriptional control of gluconeogenesis in Aspergillus nidulans. Genetics 2007, 176(1):139-150.

59. Meijer S, Panagiotou G, Olsson L, Nielsen J: Physiological characterization of xylose metabolism in Aspergillus niger under oxygen-limited conditions. Biotechnol Bioeng 2007, 98(2):462-475.

60. Felenbok B, Sequeval D, Mathieu M, Sibley S, Gwynne DI, Davies RW: The ethanol regulon in Aspergillus nidulans - characterization and sequence of the positive regulatory gene alcR. Gene 1988, 73(2):385-396. 
61. Macios M, Caddick MX, Weglenski P, Scazzocchio C, Dzikowska A: The GATA factors AREA and AREB together with the co-repressor NMRA, negatively regulate arginine catabolism in Aspergillus nidulans in response to nitrogen and carbon source. Fungal Genet Biol 2012, 49(3):189-198.

62. Wong KH, Hynes MJ, Davis MA: Recent advances in nitrogen regulation: a comparison between Saccharomyces cerevisiae and filamentous fungi. Eukaryot Cell 2008, 7(6):917-925.

63. Berger $H$, Basheer A, Böck $S$, Reyes-Dominguez $Y$, Dalik $T$, Altmann F, Strauss J: Dissecting individual steps of nitrogen transcription factor cooperation in the Aspergillus nidulans nitrate cluster. Mol Microbiol 2008, 69(6):1385-1398.

64. Schinko T, Berger $H$, Lee W, Gallmetzer A, Pirker K, Pachlinger R, Buchner I, Reichenauer T, Güldener U, Strauss J: Transcriptome analysis of nitrate assimilation in Aspergillus nidulans reveals connections to nitric oxide metabolism. Mol Microbio/ 2010, 78(3):720-738.

65. Muszkieta L, Beauvais A, Pähtz V, Gibbons JG, Anton Leberre V, Beau R, Shibuya K, Rokas A, Francois JM, Kniemeyer O, Brakhage AA, Latge JP: Investigation of Aspergillus fumigatus biofilm formation by various "omics" approaches. Front Microbio/ 2013, 4:13.

66. Wei HJ, Scherer M, Singh A, Liese R, Fischer R: Aspergillus nidulans a-1,3 glucanase (mutanase), mut $A$, is expressed during sexual development and mobilizes mutan. Fungal Genet Biol 2001, 34(3):217-227.

67. Arnaud M, Chibucos M, Costanzo M, Crabtree J, Inglis D, Lotia A, Orvis J, Shah P, Skrzypek M, Binkley G, Miyasato SR, Wortman JR, Sherlock G: The Aspergillus Genome Database, a curated comparative genomics resource for gene, protein and sequence information for the Aspergillus research community. Nucleic Acids Res 2010, 38(Database issue):D420-427.

68. Apweiler R, Martin MJ, O'Donovan C, Magrane M, Alam-Faruque Y, Alpi E, Antunes R, Arganiska J, Casanova EB, Bely B, Apweiler R, Martin MJ, O'Donovan C, Magrane M, Alam-Faruque Y, Alpi E, Antunes R, Arganiska J, Casanova EB, Bely B, Bingley M, Bonilla C, Britto R, Bursteinas B, Chan WM, Chavali G, Cibrian-Uhalte E, Da Silva A, De Giorgi M, Dimmer E, et al: Update on activities at the Universal Protein Resource (UniProt) in 2013. Nucleic Acids Res 2013, 41(D1):D43-D47.

69. Dyer PS, O'Gorman CM: Sexual development and cryptic sexuality in fungi: insights from Aspergillus species. FEMS Microbiol Rev 2012, 36(1):165-192.

70. Krijgsheld P, Bleichrodt R, van Veluw GJ, Wang F, Müller WH, Dijksterhuis J, Wosten HAB: Development in Aspergillus. Stud Mycol 2013, 74(1):1-29.

71. Paoletti M, Seymour FA, Alcocer MJC, Kaur N, Caivo AM, Archer DB, Dyer PS: Mating type and the genetic basis of self-fertility in the model fungus Aspergillus nidulans. Curr Biol 2007, 17(16):1384-1389.

72. Bayram O, Braus GH: Coordination of secondary metabolism and development in fungi: the velvet family of regulatory proteins. FEMS Microbiol Rev 2012, 36(1):1-24.

73. Ariyo B, Tamerler C, Bucke C, Keshavarz T: Enhanced penicillin production by oligosaccharides from batch cultures of Penicillium chrysogenum in stirred-tank reactors. FEMS Microbiol Lett 1998, 166(1):165-170

74. Yin W-B, Amaike S, Wohlbach DJ, Gasch AP, Chiang Y-M, Wang CCC, Bok JW, Rohlfs M, Keller NP: An Aspergillus nidulans bZIP response pathway hardwired for defensive secondary metabolism operates through aflR Mol Microbiol 2012, 83(5):1024-1034.

75. Fisch KM, Gillaspy AF, Gipson M, Henrikson JC, Hoover AR, Jackson L, Najar FZ, Waegele H, Cichewicz RH: Chemical induction of silent biosynthetic pathway transcription in Aspergillus niger. J Ind Microbiol Biotechnol 2009, 36(9):1199-1213.

76. Lerat S, Forest M, Lauzier A, Grondin G, Lacelle S, Beaulieu C: Potato suberin induces differentiation and secondary metabolism in the genus Streptomyces. Microbes Environ 2012, 27(1):36-42.

77. Baker PJ, Poultney C, Liu Z, Gross R, Montclare JK: Identification and comparison of cutinases for synthetic polyester degradation. Appl Microbiol Biotechnol 2012, 93(1):229-240.

78. Gandini A, Pascoal Neto C, Silvestre AJD: Suberin: a promising renewable resource for novel macromolecular materials. Prog Polym Sci 2006, 31(10):878-892.

79. Salazar M, Vongsangnak W, Panagiotou G, Andersen MR, Nielsen J: Uncovering transcriptional regulation of glycerol metabolism in Aspergilli through genome-wide gene expression data analysis. Mol Genet Genomics 2009, 282(6):571-586.
80. Garrido SM, Kitamoto N, Watanabe A, Shintani T, Gomi K: Functional analysis of FarA transcription factor in the regulation of the genes encoding lipolytic enzymes and hydrophobic surface binding protein for the degradation of biodegradable plastics in Aspergillus oryzae. J Biosci Bioeng 2012, 113(5):549-555.

81. Rocha ALM, Di Pietro A, Ruiz-Roldan C, Roncero MIG: Ctf1, a transcriptional activator of cutinase and lipase genes in Fusarium oxysporum is dispensable for virulence. Mol Plant Pathol 2008, 9(3):293-304.

82. Bravo-Ruiz G, Ruiz-Roldan C, Roncero MIG: Lipolytic System of the Tomato Pathogen Fusarium oxysporum f. sp. Iycopersici. Mol Plant Microbe In 2013, 26(9):1054-1067.

83. Kovalchuk A, Driessen AJM: Phylogenetic analysis of fungal $A B C$ transporters. BMC Genomics 2010, 11(1):177.

84. Andrade AC, Van Nistelrooy JGM, Peery RB, Skatrud PL, De Waard MA: The role of $A B C$ transporters from Aspergillus nidulans in protection against cytotoxic agents and in antibiotic production. Mol Gen Genet 2000, 263(6):966-977.

85. Holyoak CD, Bracey D, Piper PW, Kuchler K, Coote PJ: The Saccharomyces cerevisiae weak acid inducible ABC transporter Pdr12 transports fluorescein and preservative anions from the cytosol by an energy dependent mechanism. J Bacterio/ 1999, 181(15):4644-4652

86. Vangelatos I, Roumelioti K, Gournas C, Suarez T, Scazzocchio C, Sophianopoulou V: Eisosome organization in the filamentous ascomycete Aspergillus nidulans. Eukaryot Cell 2010, 9(10):1441-1454.

87. Kelly DE, Kraševec N, Mullins J, Nelson DR: The CYPome (cytochrome P450 complement) of Aspergillus nidulans. Fungal Genet Biol 2009, 46:S53-S61.

88. Kitazume T, Takaya N, Nakayama N, Shoun H: Fusarium oxysporum fatty-acid subterminal hydroxylase (CYP505) is a membrane-bound eukaryotic counterpart of Bacillus megaterium cytochrome P450BM3. J Biol Chem 2000, 275(50):39734-39740.

89. Vanhanen S, West M, Kroon JTM, Lindner N, Casey J, Cheng Q, Elborough KM, Slabas AR: A consensus sequence for long-chain fatty-acid alcohol oxidases from Candida identifies a family of genes involved in lipid omega-oxidation in yeast with homologues in plants and bacteria. J Biol Chem 2000, 275(6):4445-4452.

90. Hynes MJ, Murray SL, Khew GS, Davis MA: Genetic analysis of the role of peroxisomes in the utilization of acetate and fatty acids in Aspergillus nidulans. Genetics 2008, 178(3):1355-1369.

91. Reiser K, Davis MA, Hynes MJ: Aspergillus nidulans contains six possible fatty acyl-CoA synthetases with FaaB being the major synthetase for fatty acid degradation. Arch Microbiol 2010, 192(5):373-382.

92. Reiser K, Davis MA, Hynes MJ: AoxA is a major peroxisomal long chain fatty acyl-CoA oxidase required for beta-oxidation in $A$. nidulans. Curr Genet 2010, 56(2):139-150.

93. De Cremer K, Mathys J, Vos C, Froenicke L, Michelmore RW, Cammue BPA De Coninck B: RNAseq-based transcriptome analysis of Lactuca sativa infected by the fungal necrotroph Botrytis cinerea. Plant Cell Environ 2013, 36(11):1992-2007.

94. Maggio-Hall LA, Lyne P, Wolff JA, Keller NP: A single acyl-CoA dehydrogenase is required for catabolism of isoleucine, valine and short-chain fatty acids in Aspergillus nidulans. Fungal Genet Biol 2008, 45(3):180-189.

95. Pusztahelyi T, Klement É, Szajli E, Klem J, Miskei M, Karányi Z, Emri T, Kóvacs S, Orosz G, Kóvacs KL, Medzihradszky KF, Prade RA, Pócsi I: Comparison of transcriptional and translational changes caused by long-term menadione exposure in Aspergillus nidulans. Fungal Genet Biol 2011, 48(2):92-103.

96. Mathew S, Abraham TE: Bioconversions of ferulic acid, an hydroxycinnamic acid. Crit Rev Microbiol 2006, 32(3):115-125.

97. Caspi R, Altman T, Dreher K, Fulcher CA, Subhraveti P, Keseler IM, Kothari A, Krummenacker M, Latendresse M, Mueller LA, Ong Q, Paley S, Pujar A, Shearer AG, Travers M, Weerasinghe D, Zhang PF, Karp PD: The MetaCyc database of metabolic pathways and enzymes and the BioCyc collection of pathway/genome databases. Nucleic Acids Res 2012, 40(D1):D742-D753.

98. Tamayo EN, Villanueva A, Hasper AA, de Graaff LH, Ramón D, Orejas M: CreA mediates repression of the regulatory gene $x \ln R$ which controls the production of xylanolytic enzymes in Aspergillus nidulans. Fungal Genet Biol 2008, 45(6):984-993. 
99. Gygi SP, Rist B, Gerber SA, Turecek F, Gelb MH, Aebersold R: Quantitative analysis of complex protein mixtures using isotope-coded affinity tags. Nat Biotechnol 1999, 17(10):994-999.

100. Hinnebusch AG: Translational regulation of GCN4 and the general amino acid control of yeast. Annu Rev Microbiol 2005, 59:407-450.

101. Beyer A, Hollunder J, Nasheuer HP, Wilhelm T: Post-transcriptional expression regulation in the yeast Saccharomyces cerevisiae on a genomic scale. Mol Cell Proteomics 2004, 3(11):1083-1092.

102. Laloo B, Simon D, Veilla V, Lauzel D, Guyonnet-Duperat V, Moreau-Gaudry F, Sagliocco F, Grosset C: Analysis of post-transcriptional regulations by a functional, integrated, and quantitative method. Mol Cell Proteomics 2009, 8(8):1777-1788.

103. Martins I, Garcia H, Varela A, Núñez O, Planchon S, Galceran MT, Renaut J, Rebelo LPN, Silva Pereira C: Investigating Aspergillus nidulans secretome during colonisation of cork cell walls. J Proteomics 2014, 26(98):175-188.

doi:10.1186/1471-2164-15-613

Cite this article as: Martins et al:: Elucidating how the saprophytic

fungus Aspergillus nidulans uses the plant polyester suberin as carbon source. BMC Genomics 2014 15:613.

\section{Submit your next manuscript to BioMed Central and take full advantage of:}

- Convenient online submission

- Thorough peer review

- No space constraints or color figure charges

- Immediate publication on acceptance

- Inclusion in PubMed, CAS, Scopus and Google Scholar

- Research which is freely available for redistribution 